\title{
A systematic evaluation of Flow Field Flow Fractionation and single-particle ICP-MS to obtain the size distribution of organo-mineral iron oxyhydroxide colloids
}

Claudia Moens $^{\mathrm{a} 1}$, Nadia Waegeneers ${ }^{\mathrm{b}}$, Andreas Fritzsche ${ }^{\mathrm{c}}$, Peter Nobels ${ }^{\mathrm{d}}$, Erik Smolders ${ }^{\mathrm{a}}$

a Department of Earth and Environmental Sciences, KU Leuven, Kasteelpark Arenberg 20 bus 2459, 3001 Leuven, Belgium; claudia.moens@kuleuven.be; erik.smolders@kuleuven.be

b Service Trace Elements \& Nanomaterials, Sciensano, Leuvensesteenweg 17, 3080 Tervuren, Belgium; nadia.waegeneers@sciensano.be

c Institut für Geowissenschaften, Friedrich-Schiller-Universität, Burgweg 11, 07749 Jena, Germany; fritzsche-andreas@web.de

d Department of Environmental Sciences, Wageningen University \& Research, PO box 47, 6700AA Wageningen, The Netherlands; peter.nobels@wur.nl

1 Corresponding author: claudia.moens@kuleuven.be; phone: +32 16377044

\section{Abstract}

Colloidal iron(III) oxyhydroxides (FeOx) are important reactive adsorbents in nature. This study was set up to determine the size of environmentally relevant FeOx colloids with new methods, i.e. Flow Field Flow Fractionation (FIFFF-UV-ICP-MS) and single-particle ICP-MS/MS (sp-ICP-MS) and to compare these with standard approaches, i.e. dynamic light scattering (DLS), nanoparticle tracking analysis (NTA), microscopy (TEM), membrane filtration, centrifugation and dialysis. Seven synthetic nano- and submicron FeOx with different mineralogy and coating were prepared and two soil solutions were included. The FIFFF was optimized for Fe recovery, yielding $70-90 \%$. The FIFFF determines particle size with high resolution in a $1 \mathrm{mM} \mathrm{NH}_{4} \mathrm{HCO}_{3}(\mathrm{pH} 8.3)$ background and can detect Fe-NOM complexes $<5$ $\mathrm{nm}$ and organo-mineral FeOx particles ranging 5-300 nm. The sp-ICP-MS method had a size detection limit for FeOx of about 32-47 nm. The distribution of hydrodynamic diameters of 
goethite particles detected with FIFFF, NTA and DLS were similar but the values were twice as large as the Fe cores of particles detected with sp-ICP-MS and TEM. Conventional fractionation by centrifugation and dialysis generally yielded similar fractions as FIFFF but membrane filtration overestimated the large size fractions. Particles formed from Fe(II) oxidation in the presence of NOM showed strikingly smaller organo-mineral Fe-Ox colloids as the NOM/Fe ratio increased. The soil solution obtained with centrifugation of an acid peat was dominated by small $(<30 \mathrm{~nm}$ ) Fe-OM complexes and organo-mineral FeOx colloids whereas that of a mineral pH neutral soil mainly contains larger $(30-200 \mathrm{~nm})$ Fe-rich particles. The FIFFF-UV-ICP-MS is recommended for environmental studies of colloidal FeOx since it has a wide size detection range, it fractionates in an environmentally relevant background ( $1 \mathrm{mM}$ $\mathrm{NH}_{4} \mathrm{HCO}_{3}$ ) and it has acceptable element recoveries.

\section{Highlights:}

- FIFFF distinguishes between Fe-OM complexes and organo-mineral FeOx colloids

- Size detection limit of sp-ICP-MS/MS compromises sizing of FeOx colloids

- $\mathrm{Fe}(\mathrm{II})$ oxidation with OM results in decreased colloid size with increasing OM/Fe ratio

- Small Fe-OM colloids prevail in peat soil and larger Fe-rich colloids in mineral soil

\section{Keywords:}

Flow Field Flow Fractionation; Single-particle inductively coupled plasma-mass spectrometry; Triple quadrupole mass spectrometry; organo-mineral iron oxyhydroxide colloids; Particle size distribution; Multimethod comparison

\section{Introduction}

Iron (Fe) is one of the most common elements on earth. At the earth surface, Fe is present as $\mathrm{Fe}$ (III) that hydrolyses and precipitates as poorly soluble oxyhydroxides (FeOx) with a wide 
range of potential crystalline structures. These FeOx minerals chiefly form by weathering of Fe(II) silicates or by oxidation of soluble Fe(II). Upon oxidation, amorphous Hydrous Ferric Oxides (HFO) are initially formed [1] that transform to more crystalline Fe minerals such as ferrihydrite $\left(\mathrm{Fe}_{2} \mathrm{O}_{3} \bullet 2 \mathrm{H}_{2} \mathrm{O}\right)$, goethite $(\mathrm{FeOOH})$ and hematite $\left(\mathrm{Fe}_{2} \mathrm{O}_{3}\right)$ [2]. All FeOx minerals have highly reactive surface on which the negatively charged Natural Organic Matter (NOM) sorbs in the environment [3]. The FeOx minerals can be present as particulates and as colloids, the latter more readily in association with NOM or inorganic anions (e.g. phosphate) that could inverse the slightly net-positive surface charge of $\mathrm{FeOx}$ at circumneutral $\mathrm{pH}$ to highly netnegative surface charge resulting in increased environmental stability of the FeOx colloids.

The $\mathrm{Fe}^{3+}$ ion may form mononuclear complexes with NOM, this complexation suppresses the hydrolysis and polymerization of Fe [4], [5]. At high C/Fe, Fe will be complexed with NOM, at low C/Fe the complexation capacity of NOM is exceeded and FeOx form. The hydrolysis and polymerization of $\mathrm{Fe}^{3+}$ is promoted at higher $\mathrm{pH}$ and becomes more dominant than NOM complexation. Therefore, Fe-NOM complexes typically dominate the Fe speciation over FeOx in acid freshwaters and soils provided that sufficient NOM is present [4], [6].

The Fe-OM complexes and organo-mineral FeOx can occur as natural colloids with size ranges down to a few nm [1]. Colloidal FeOx are strong adsorbents for organic matter, oxyanions and trace metal cations and, hence, have a large environmental effect as mobile carrier of nutrients and contaminants in aquatic and terrestric environments [7]. Non-settling FeOx colloids may be mobile in aquatic environments and through macropores in soils and sediments. The mobility of those FeOx are of high interest to assess and predict the temporal and spatial shifts in reactivity within ecosystems. The determination of the colloidal size is crucial for predicting colloid mobility. Small colloids move predominantly under Brownian 
motion and are less impeded by straining in saturated porous media [8]. However, the large surface reactivity of Fe colloids makes size characterization challenging because large fractions of Fe can be lost during treatment. So far, the traditional methods available for fractionation and sizing of natural nano- and submicron particles are (ultra)filtration, centrifugation, dialysis, microscopy, size exclusion chromatography and light-scattering based techniques, e.g. Dynamic Light Scattering (DLS) and Nanoparticle Tracking Analysis (NTA).

Several fractionation techniques showed that a considerable fraction of $\mathrm{Fe}$ in $\mathrm{pH}$ neutral streams has colloidal dimensions, either as Fe-NOM complexes or FeOx colloids [6], [9], [10]. Lofts et al. [6] used dialysis to separate truly dissolved and colloidal Fe and observed that FeOx dominate the Fe speciation ( $<0.45 \mu \mathrm{m}$ filtrate) in $\mathrm{pH}$ neutral streams, Fe-NOM complexes are about an order of magnitude lower in concentration. Iron was also identified as a key constituent of the coarse colloidal fraction (100 nm-1200 nm) examined in about 50 Belgian streams. On average, $70 \%$ of the Fe in the filtered $(<1.2 \mu \mathrm{m})$ fraction is present in this size range [10]. Although filtration is often applied in environmental studies, this technique is known to be prone to considerable artefacts for colloids because they can interact with the filter, induce pore clogging or aggregate due to the increased concentrations of the retained particles at the membrane surface [11]. Size exclusion chromatography (SEC) has been criticized because analysis suffer from interactions of the analyte components with the column packing material [12] and eluens with environmental irrelevant high ionic strengths are used. In addition, the size range is limited to the low nanometer range, a study on surface water colloids showed the inability of SEC to differentiate between NOM and Fe organomineral colloids [13]. 
Field Flow Fractionation (FFF) and single-particle ICP-MS (sp-ICP-MS) are emerging techniques in the field of nanoparticle characterization [14]. The Flow Field Flow Fractionation technique [15] coupled online to ICP-MS, UV-VIS and fluorescence detectors is increasingly used to study Fe oxide nanoparticles, in synthetic solutions [16]-[18] as well as naturally occurring Fe colloids. This technique has given valuable information about colloidal carriers and associated elements in streams [19]-[22], groundwater [23], [24] and soil extracts [25], [26]. Several of these FIFFF studies revealed that Fe is mostly present in the small $(<3 \mathrm{~nm})$ organic Cdominated fraction as Fe-OM complexes and in larger size fractions (> $10 \mathrm{~nm}$ ) as organomineral FeOx colloids. Flow Field Flow Fractionation is a separation technique, but particle size can be deduced applying FFF theory or by calibration with spherical particles with certified size. The obtained size is a hydrodynamic diameter, and represents the diameter of a hard sphere with the same diffusion properties. However, natural colloids are unlikely hard spheres as assumed in FFF theory and there are no certified Fe particles available for calibration, which might result in size bias [27]. In addition, both size calibration methods rely on the assumption that ideal behavior occurs in the FFF channel, but artefacts can occur during separation, like channel overloading, steric elution, shape selective retention and particle-membrane interactions [28]. Therefore, validation of this approach by comparison with other sizing techniques is essential. The proposed methodology should then be tested on a range of particles that might occur in the environment e.g. using simplified systems of synthetic FeOx-NOM particles with experimentally varied properties to determine the suitability of the FIFFF method. In addition, analyte recovery receives little attention in environmental studies using FIFFF, a parameter that needs attention for FeOx given its high surface reactivity. 
Single-particle ICP-MS analysis [29] performed on environmental matrices mostly deal with engineered nanoparticles, e.g. $\mathrm{Ag}, \mathrm{Au}, \mathrm{Ti}$ but the technique is only rarely applied to characterize natural colloids. Degueldre and Favarger [30] used sp-ICP-MS to characterize model colloids, amongst others goethite, and a natural clay in water. Gomez-Gonzales et al. [31] detected scorodite $\left(\mathrm{FeAsO}_{4} \cdot 2 \mathrm{H}_{2} \mathrm{O}\right)$ colloids based on As in mine leachates. Measuring FeOx colloids with sp-ICP-MS is challenging due to the polyatomic interference of $\mathrm{ArO}^{+}$on $\mathrm{m} / \mathrm{z}=56$ [32] [30]. We previously sized model FeOOH colloids with sp-ICP-MS analysis with ICP-MS/MS and obtained a lower detection threshold of about $40 \mathrm{~nm}$ [33]. It is yet unclear how this interference compromises sizing of environmentally relevant FeOx particles.

The objective of this study is to evaluate the potential of sp-ICP-MS and FIFFF-UV-ICP-MS to determine the size distribution of environmentally relevant FeOx colloids. The FIFFF method likely yields the lowest size detection threshold but may have poor Fe recoveries due to its interaction with the membrane and method optimization is required. Seven synthetic nanoand submicron FeOx were prepared with contrasting mineralogy, Fe/NOM composition, coating and size. In addition, the techniques were applied on soil solutions of two contrasting soils to include analytical complex samples. First, both sp-ICP-MS and FIFFF-UV-ICP-MS methods were optimized for low size detection limits, reproducibility and recoveries. Second, the particle size distributions obtained with these two more recent methods are compared with more conventional fractionation and sizing techniques, i.e. DLS, NTA, TEM, membrane filtration, centrifugation and dialysis in a matrix approach. This multimethod comparison assists in interpretation of the obtained spherical diameters because environmental FeOx colloids are mostly heterogeneous, non-spherical particles and have broad size distributions.

\section{Materials and Methods}




\subsection{Synthetic and natural colloids}

Seven different synthetic FeOx colloids were used (Table 1) to obtain a range of contrasting composition and mineralogy that may be expected in the environment. The particle set consists of two samples of goethite (Gt), one sample of ferrihydrite (Fh) and four samples of Hydrous Ferric Oxide (HFO). Particles were prepared with either NOM or arsenate (As) as coating to stabilize the particles by surface charge reversion. In the environment, the FeOx particles are mainly associated with NOM and oxyanions such as phosphate $(P)$ at their surface; here As was used rather than P to obtain better ICP-MS detection limits on the FIFFF fractions of the oxyanion sorbed to the surface, data that are not reported here.

Goethite (Gt) particles were purchased from Sigma Aldrich (20 wt\% in water). Ferrihydrite (Fh) was synthesized from $\left(\mathrm{Fe}\left(\mathrm{NO}_{3}\right)_{3}\right)$ according to Burleson and Penn [34] without microwave anneal step to produce ferrihydrite with average primary particle size of about $3 \mathrm{~nm}$. The Gt and Fh have been characterized with DLS, XRD, FTIR and SEM in a previous study and with spICP-MS (only Gt).[35]. Suwannee River Natural Organic Matter (SRNOM, International Humic Substances Society, 2R101N) or arsenate (As, added as $\mathrm{Na}_{2} \mathrm{HAsO}_{4}$ ) was added to the goethite colloids (referred to as Gt-NOM and Gt-As respectively) and As was added to ferrihydrite to increase stability at $\mathrm{pH} 6$. The HFO was prepared by coprecipitation of Fe(II) in presence of SRNOM. The Fe salt $\left(\mathrm{FeSO}_{4} .7 \mathrm{H}_{2} \mathrm{O}\right)$ was added to SRNOM containing test solution and was subsequently allowed to oxidize for $48 \mathrm{~h}$ at $20^{\circ} \mathrm{C}$ in darkness [36]. The test solutions contained varying amounts of SRNOM (nominal molar C/Fe ratios 100, 45, 5 end 2.5) relevant for stream water samples. Increasing C/Fe ratios are expected to yield increasing fractions of very small organic mononuclear Fe colloids. Chemical modelling (Visual Minteq) indicates that all Fe can be complexed as mononuclear complexes at a molar C/Fe ratio of 100 . 
All synthetic samples were dissolved in $5 \mathrm{mM}$ 2-(N-morpholino)ethanesulfonic acid (MES buffer) adjusted to $\mathrm{pH} 6.0$ with $\mathrm{NaOH}$. Soil solutions were obtained with centrifugation from two soil samples with contrasting properties (Table 2), an organic matter rich soil with low pH from a managed grassland (Rhytalog, UK $53^{\circ} 06^{\prime} 02^{\prime \prime} \mathrm{N}, 3^{\circ} 09^{\prime} 41^{\prime \prime} \mathrm{W}$ ) and a $\mathrm{pH}$ neutral mineral

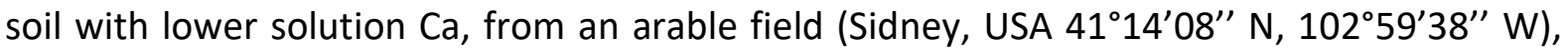
referred to as SS-Rhy and SS-Sid respectively. The air dried and sieved soils $(<2 \mathrm{~mm})$ were wetted with demineralized water and incubated for two days (at solid/liquid ratio $(\mathrm{g} / \mathrm{g}$ ) of 1.5 for Rhytalog and 2.5 for Sidney soil) in order to equilibrate the added water with the soil with regularly mixing to avoid reducing conditions. Soil solution was extracted by centrifugation at $2000 \mathrm{~g}$ for $15 \mathrm{~min}$. The soil sample was placed in a double chamber setup, which consists of a compartment that contains the soil and a second compartment underneath where soil solution is collected due to the applied centrifugal force. A membrane filter of $0.45 \mu \mathrm{m}$ served as separation between the soil and soil solution compartments. In this set-up, the filter could not completely avoid some larger particles to enter the soil solution compartment, but these particles quickly settled out and no additional centrifugation/filtration step was done prior to sample composition and particle size analysis.

For all synthetic samples and soil solutions, the total element concentrations were determined in unfiltered samples with Inductively Coupled Plasma Mass Spectrometry (ICPMS, Agilent 7700X) after aqua regia digestion (1 mL of the colloidal solution, $1.5 \mathrm{~mL}$ of $\mathrm{HCl}$ and $0.5 \mathrm{~mL}$ of $\mathrm{HNO}_{3}$ ) for $2 \mathrm{~h}$ at $140{ }^{\circ} \mathrm{C}$. Soil $\mathrm{pH}$ was measured in $0.01 \mathrm{M} \mathrm{CaCl}_{2}$ (soil/solution ratio $1 / 5)$ after $1 \mathrm{~h}$ shaking and 30 min settling. Total carbon (C) was measured by ignition with a vario Max N/CN analyser (Elementar Analysensysteme $\mathrm{GmbH}$ ). The carbonate- $\mathrm{C}$ was measured from pressure increase after acidification $(\mathrm{HCl})$ to the soil in closed containers 
(release of $\mathrm{CO}_{2}$ from organic matter was minimized with reducing agent $\mathrm{FeSO}_{4}$ ) [37]. Organic $\mathrm{C}$ was determined as the difference between Total $\mathrm{C}$ and $\mathrm{CaCO}-\mathrm{C}$. The $\mathrm{Feo}_{\mathrm{o}}$ and $\mathrm{Alo}$ are ammonium oxalate-extractable concentrations, which is a measure of the amorphous Fe and Al oxyhydroxides in soil [38]. The DOC in soil solutions was measured with a TOC-analyser.

Beside FIFFF and sp-ICP-MS analysis, all synthetic samples are fractionated with dialysis, membrane filtration and centrifugation, hydrodynamic diameters are determined with NTA and DLS and particles are characterized with TEM. The methodology of these measurements are described in supplementary material. For each particle type, all size measurements were performed on the same batch as short as possible after synthesis. Centrifugation, dialysis, membrane filtration, DLS, FIFFF and Sp-ICP-MS were carried out within one week after synthesis. Due to practical reasons, TEM and NTA analysis were performed within one month after synthesis. Stock solutions were stored in the fridge and DLS and sp-ICP-MS did not record important change in size during this period. Storage affected size more for natural samples (i.e. soil solutions) than for synthetic samples, after a few days some precipitation was observed in the natural samples, not in the synthetic samples. Therefore size fractionation was performed on the soil solutions within two days and DLS and NTA was not performed on these sample. TEM analysis were also not performed because elemental detection was not possible to distinguish FeOx colloids from other soil colloids.

\subsection{Instrumentation Flow Field Flow Fractionation (FIFFF-UV-ICP-MS) analysis}

Colloids were analyzed with Flow Field Flow Fractionation (FIFFF) (AF2000, Postnova Analytics) online coupled with an UV/VIS detector (diode array detector, SPD-20A Postnova Analytics) and ICP-MS (Agilent 7700X). The Fe concentration was monitored on the ${ }^{56} \mathrm{Fe}$ isotope line. The colloids in this study exhibited sizes exceeding the practicable range to be 
measured with one single method using constant cross-flow rate. Therefore different procedures were compared in which spacer thickness and cross-flow rate were varied and tested to obtain maximal Fe recoveries. The settings of the FIFFF-UV-ICP-MS system and the method used for each sample is indicated in Table S1. FFF theory was used to convert retention time $\left(T_{r}\right)$ into hydrodynamic diameters $\left(D_{h}\right)$. The linear approximation $R=6 \lambda$ with retention ratio $R$, obtained from the fractogram and retention parameter $\lambda$, containing FFF parameters and particle $D_{h}$ was used [15]. The FIFFF methods were checked by comparing elution profiles from commercially available polystyrene standards of defined diameter (20, 50, 100 and $500 \mathrm{~nm}$ ) with the theoretical elution time. The reference particles are dispersed in $0.2 \%$ Novachem (surfactant) and $0.02 \%$ Novachem was added to the carrier. For methods 3 and 4, used to separate the relatively large particles (Table S1), the regression line between $D_{h}$ and retention time was linear $\left(R^{2}>0.99\right)$ and the slopes agree (less than $15 \%$ difference) with FIFFF theory (data not shown). For methods 1 and 2, used to separate small particles, only the $20 \mathrm{~nm}$ polystyrene standard is covered within the separation range for calibration and the three samples (HFO 110/45/5 C/Fe) are therefore extrapolations to lower retention times. The linear relation used to calculate particle diameter is valid for retention time of the analyte $T_{r}$ that is sufficiently long compared to an unretained component $T_{0}\left(T_{r}>6 * T_{0}\right)$ [15], which is shown to be valid for small particles [19], [21]. In this study, this condition is met, the sample peaks eluted within the range $T_{r}=10-50 T_{0}$, except for one sample (HFO $\left.110 \mathrm{C} / \mathrm{Fe}\right)$, which has a relatively low retention with $\mathrm{T}_{r}=5 \mathrm{~T}_{0}$. Applying FFF theory on early eluting analytes results in overestimation of particle size.Because the fate of Fe is ultimately linked with that of organic matter, a UV/VIS detector monitored the absorbance at wavelength $254 \mathrm{~nm}$, which is a proxy for aromatic organic matter [39]. The absorbance was corrected afterwards for interference of $\mathrm{Fe}(\mathrm{III})$ [40]. All samples were analyzed in duplicate. 


\subsection{Instrumentation Single-particle ICP-MS/MS}

An ICP-MS/MS (Agilent 8800) was used for data acquisition in time-resolved analysis mode. Ammonia $\left(\mathrm{NH}_{3}\right)$ was thereby used as reaction gas to reduce interference by argon oxide $\left(\mathrm{ArO}^{+}\right)$ at $\mathrm{m} / \mathrm{z} 56$ in $\mathrm{MS} / \mathrm{MS}$ mode [41]. In this study the $\mathrm{NH}_{3}$ flow rate was optimized to a give high net $\mathrm{Fe}^{+}$signal with reduced argon oxide interference. To increase the sensitivity of the sp-ICPMS analyses, instrument tuning was optimized for ${ }^{56} \mathrm{Fe}$ by adjusting, amongst others, sample depth and carrier gas flow rate. The instrument parameters and operational conditions are given in Table S2. The transport efficiency was determined daily according to the particle frequency method [42] by means of the reference material NIST-8012 (gold nanoparticles, nominal size $30 \mathrm{~nm})$.

The single particle calculation spreadsheet described by Peters et al. [43] was used to calculate particle size distributions and particle number or mass concentrations. The particle diameter is obtained from the particle mass assuming a spherical geometry, referred to as spherical volume equivalent diameter $\left(D_{v}\right)$. To discriminate between particle events and remaining $\mathrm{ArO}^{+}$interference, an iterative algorithm was applied on the data obtained from ultrapure water as described by Tuoriniemi et al. [44]. The Fe colloid samples were diluted with ultrapure water for sp-ICP-MS measurement. An appropriate dilution was thereby determined after a range finding test (illustrated in figure S1). Ideally, the dilution should result in a proportionally changing number of detected particles (between 200 and 2500 to have an adequate amount of single-particle events) and a constant particle size. The samples for final sp-ICP-MS measurement were diluted in duplicate (synthetic samples) or triplicate (soil solutions) and two replicate measurements for each dilution were performed yielding four replicates (synthetic samples) or six (soil solutions) for statistical analysis. 


\subsection{Statistical analysis}

Statistical analysis was performed with JMP Pro 13 (SAS), all hypothesis were tested at the 0.05 significance level. The Tukey HSD test for multiple comparison was used to compare for each synthetic sample the size distributions among the different sizing techniques (Table 4) and to compare the size difference between particles according to each sizing technique (Table S4). No statistical analysis was applied on the size distributions of particles measured in the soil solutions or on the size fractionations obtained with membrane filtration, centrifugation and dialysis.

\section{Results}

\subsection{General properties of the synthetic particles based on standards approaches}

Addition of NOM or As to Gt and Fh at pH 6 resulted in a negative zetapotential (Table 1). To the Fh and Gt samples arsenate was added in excess post-synthesis to include the influence of polarity of surface charge on method performance. The dialysis indicates that Fh sorbs As at higher molar As/Fe ratio than $\mathrm{Gt}, 73 \%$ of As is truly dissolved in the Fh sample and $90 \%$ in the Gt sample, the remaining part being sorbed on the particles which is consistent with a larger surface site density of ferrihydrite compared to goethite [45]. The HFO samples contained varying amounts of SRNOM (nominal molar C/Fe ratios 100, 45, 5 end 2.5). At higher molar $\mathrm{C} / \mathrm{Fe}$ ratio ( $\mathrm{C} / \mathrm{Fe} 1)$, an unstable particle solution was formed at $\mathrm{pH} 6$ suggesting that at this molar ratio not enough negative charge was added to stablilize the FeOx colloids at $\mathrm{pH} 6$.

Needle shaped colloids are observed in the TEM images of Gt (Figure 1) The Gt colloids have a median aspect ratio of about 4 , a long axis length between $\sim 50-110 \mathrm{~nm}$ and a short axis 
length between $15-30 \mathrm{~nm}\left(10^{\text {th }}\right.$ and $90^{\text {th }}$ percentile of number-based distribution of $>1000$ particles in the Gt-NOM sample). Single particles cannot clearly be distinguished in the TEM images of Fh and $\mathrm{HFO}$, but the particle dimensions range $\mathrm{HFO}<\mathrm{Fh}<\mathrm{Gt}$ (Figure 1).

Table 3 gives the fractionation obtained with membrane filtration (MF), centrifugation (CF) and dialysis. Membrane filtration and centrifugation confirm the size ranking determined with TEM, i.e. HFO<Fh<Gt (Table 3). CF indicates the presence of larger particles in Gt-As compared to Gt-NOM, for instance in Gt-NOM only $17 \%$ of total Fe is larger than $100 \mathrm{~nm}$, but $41 \%$ in GtAs. Larger FeOx particles are formed in $\mathrm{Fe}^{2+}$ oxidized samples with lower NOM concentrations as indicated by both MF and CF : for instance, the $<25 \mathrm{~nm}$ filtered Fe fraction of the HFO samples decreased from $>80 \%$ to $<15 \%$ when increasing the $\mathrm{C} / \mathrm{Fe}$ ratio in the tested range. Dialysates $(10-12 \mathrm{kDa})$ of the samples contain $<5 \%$ of total Fe, except for the two highest NOM-containing HFO (HFO 110 C/Fe: 20\% Fe; HFO 45 C/Fe: 10\%), illustrating that these samples contain very small particles, about $3 \mathrm{~nm}$ in diameter [46].

The NTA and DLS both showed the same trend in $D_{h}$, i.e. they ranked Fh > Gt-NOM (Table S4). The size distribution of Fh is very polydisperse as indicated by the wide PDI measured with DLS ( 0.3). The DLS and NTA could not quantify the size distribution of the HFO samples. With DLS analysis, the scattering intensities were below the quantification limit (Table 4), indicating either the absence or too small colloids with respect to their concentration. Only for HFO 2.5 $\mathrm{C} /$ Fe the scattering intensities exceeded this quantification limit marginally and a bimodal distribution was measured with $D_{h} 142$ and $D_{h} 2281 \mathrm{~nm}$, the former contributing 35\% of the total scattering intensity. This equals to a higher concentration of the smaller colloids compared to the larger colloid fraction. The NTA measured relatively large $D_{h}$ in the HFO samples ( $D_{h} 50$ between $\left.80-100 \mathrm{~nm}\right)$ and there was no trend in particle size and concentration 
depending on the NOM content. For a reliable calculation of $D_{h}$, the required amount of valid tracks is obtained (> 200) but, similarly to DLS, the particle concentration is relatively low for reliable analysis ( 4-15 particles/frame). The low particle concentration detected suggest that aggregates are measured rather than the targeted small particles.

\subsection{Flow Field Flow Fractionation (FIFFF-UV-ICP-MS) on synthetic FeOx colloids}

3.2.1 Method optimization. Initial FIFFF runs used FeOx colloids without NOM or oxyanions and yielded virtually no elution; Fe recoveries were $<10 \%$. This is attributed to their retention on the membrane, witnessed by large marks (stains) composed of positively charged FeOx on the negatively charged polyethersulfone membrane. Environmental FeOx colloids occur with surface adsorbed NOM or oxyanions and these yielded higher recoveries in FIFFF (> 70\%), similar as in the environment.

3.2.2 Sample recovery. The Fe recovery was optimized using low cross-flow rates $(<1.5 \mathrm{~mL}$ $\left.\mathrm{min}^{-1}\right)$ and relatively low carrier ionic strength $\left(1 \mathrm{mM} \mathrm{NH}_{4} \mathrm{HCO}_{3}\right)$. The recovery is calculated by integrating the Fe concentration peak profiles between the void peak and the peak that might occur when cross-flow is switched to zero at the end of the run and is expressed relative to the peak area of a sample run without cross-flow. The carrier composition was suggested from previous work on natural colloids [27]. The relatively low carrier ionic strength yields high Fe recovery without marked decrease in retention time for the sample load used in the synthetic samples (Figure S2). Each sample was run with different methods (spacer thickness and cross-flow rate varied) and particle size was calculated. The different methods yielded similar size but showed a dramatic effect of cross-flow on Fe recovery (Table S3) and a cross-

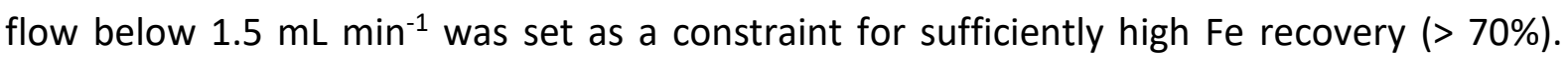
Therefore, increased channel thickness rather than high cross-flow rates are applied here to 
increase retention of small HFO particles. Under the applied conditions, the Fe recovery ranges between $70-95 \%$. The unrecovered part is mainly attributed to loss of particles on the membrane (or on tubings), not to loss of truly dissolved Fe ( $<1 \mathrm{kDa})$ because Fe has a very low free ion concentration which could have passed the $1 \mathrm{kDa}$ PES membrane. The lowest recovery in synthetic samples was observed in Fh and Gt-As which are electrostatically stabilized due to As sorption, in contrast NOM stabilized particles have a higher recovery probably related to additional sterical repulsion between particle and membrane provided by the NOM molecules. The recovery in the SS-Sid was lowest among all samples (70\%) and additional Fe was eluted (not included in the recovery) when the cross-flow was stopped (>200 nm), suggesting that this sample contained large particles that were not separated in the FIFFF (Figure 3).

3.2.3 Particle size distributions. The different Fe colloids span a wide size range with median hydrodynamic diameter $\left(D_{h}\right)$ of a few nanometer to about hundred $n m$ and broad size distributions up to about $300 \mathrm{~nm}$ (Table 4). The size distributions obtained with FIFFF are mass-based distributions. The fractograms of HFO illustrate that FIFFF can separate small particles with high resolution (Figure 2). The HFO particles produced by $\mathrm{Fe}^{2+}$ salt oxidation are increasingly large as the NOM dose decreases. At high C/Fe ratio, both the Fe and absorbance peak coincide at small particle size $(<5 \mathrm{~nm})$, in the lower $\mathrm{C} /$ Fe sample, two absorbance peaks occurs. One peak at small particle size $(<5 \mathrm{~nm})$ and a second peak coinciding with the Fe peak shifted towards larger size (about $10 \mathrm{~nm}$ ). The NOM and As additionally affected the sizes: GtNOM and Gt-As have the same Fe core, but the arsenate anion as a stabilization agent yields a significantly larger median $D_{h}$ and wider size distribution than NOM $\left(D_{h} 10, D_{h} 50\right.$ and $D_{h} 90$ of Gt-NOM is significantly smaller than the $D_{h} 10, D_{h} 50$ and $D_{h} 90$ of Gt-As). Overall, the 
particles can be ranked in size $\mathrm{HFO}<\mathrm{Fh}<\mathrm{Gt}-\mathrm{NOM}<\mathrm{Gt}-\mathrm{As}$ (Table S4), confirming trends observed with TEM, filtration and centrifugation.

\subsection{Single-particle ICP-MS/MS on synthetic FeOx colloids}

3.3.1 Method optimization. To measure FeOx colloids in single-particle ICP-MS mode, the background signal produced by the polyatomic interference of $\mathrm{ArO}^{+}$on $\mathrm{m} / \mathrm{z}=56$ needs to be overcome to decrease the size detection limit. Stephan and Hineman previously demonstrated the suitability to use $\mathrm{NH}_{3}$ as reaction gas in sp-ICP-MS analysis through the analysis of standard $\mathrm{Au}$ and $\mathrm{Fe}$ nanoparticles in deionized water [41]. Any unwanted/unexpected reactions of the highly reactive $\mathrm{NH}_{3}$ with plasma or matrix elements in single quad ICP-MS instruments can be eliminated in more complex samples by working in MS/MS mode [47]. First the sp-ICP-MS method was optimized by comparing the intensities at $\mathrm{m} / \mathrm{z} 56$ (Q1: $\mathrm{m} / \mathrm{z} 56$ - Q2: scanned) in ultrapure water, 2 and $10 \mu \mathrm{g} \mathrm{L}^{-1} \mathrm{Fe}$ using "No gas" in the collision/reaction cell, $\mathrm{He}$ or $\mathrm{NH}_{3} / \mathrm{He}$. The best results in terms of low background signal in ultrapure water and high sensitivity was observed with $\mathrm{NH}_{3} / \mathrm{He}$ and the product ion scans indicated that no unwanted reaction products with $\mathrm{NH}_{3}$ were formed. The $\mathrm{NH}_{3}$ flow rate was optimized at $0.2 \mathrm{ml} \mathrm{min}^{-1}$ which gave a high net $\mathrm{Fe}^{+}$signal, although the $\mathrm{ArO}^{+}$was thereby not completely removed. At higher flow rates, however, there was a loss of Fe signal due to reaction of $\mathrm{Fe}$ with $\mathrm{NH}_{3}$ with formation of $\mathrm{Fe}\left(\mathrm{NH}_{3}\right)_{2}$ at $\mathrm{m} / \mathrm{z} 90$ resulting in decreased instrument sensitivity (Figure S3).

A limit for particle detection has to be set to differentiate particles from background signal related with the remaining $\mathrm{ArO}^{+}$interference. Here the iterative algorithm with the mean + $n \sigma$ threshold was applied [44]. Figure S4 illustrates that a limit for particle detection set at a threshold of mean $+3 \sigma$ still included a significant number of false positive events in ultrapure 
water (80 events), while a mean $+4 \sigma$ threshold resulted in less than 20 particle events in ultrapure water measured before and in-between Fe colloid samples, which was deemed acceptable.

Figure S4 shows that the frequency distribution of the particle-related ${ }^{56} \mathrm{Fe}$ signal in the $\mathrm{Fe}$ colloid samples overlaps with the background signal determined in ultrapure water. The overlap of the ${ }^{56} \mathrm{Fe}$ signal in the Fe colloid samples with the background signal may indicate the presence of ionic Fe and/or particles with a size below the size detection limit. Ionic background can, however, be excluded since Fe(III) has very low truly dissolved concentration as confirmed with dialysis (Table 3 ). In addition, in the highly diluted samples the intensity generated if all Fe would be present as ionic Fe is only 5000 cps, which is almost tenfold lower than the mean $+4 \sigma$ threshold intensity. The same signal intensity as in the blank was applied as limit for particle detection in the samples. This means that only data above mean $+4 \sigma$ are considered as particle events and the data below this threshold are removed. The average background signal is subtracted from particles events, which resulted in size detection limits varying from 32 to $47 \mathrm{~nm}$ assuming spherical goethite particles with particle density $4 \mathrm{~g} / \mathrm{cm}^{3}$. The size detection limit depends on the daily variable nebulization efficiency, sample flow rate and calibration slope for sp-ICP-MS. Hence, with this approach using MS/MS technology, the size detection limit (SDL) for instance for goethite is reduced compared to single quad ICP-MS with collision cell technology (SDL $85 \mathrm{~nm}$ ) or using normal mode (SDL $\sim 170 \mathrm{~nm}$ ) [32] or when the low abundant ${ }^{57} \mathrm{Fe}$ isotope is measured (SDL $200 \mathrm{~nm}$ ) [30].

3.3.2 Sample recovery. The mass fraction of Fe detected as particles compared to the corresponding aqua regia digested sample is $26 \pm 0.8 \%$ for the Gt-NOM sample and $48 \pm 1.8 \%$ for the GT-As sample. Only $9 \pm 0.6 \%$ of Fe in the Fh sample could be recovered under 
particulate form by sp-ICP-MS analysis. This low recovery might indicate that a large fraction of the particles is smaller than the sp-ICP-MS detection limit, which means that the spherical volume equivalent diameter $D_{v}$ derived from the detected particles in sp-ICP-MS largely overestimates that of the original population.

3.3.3 Particle size distributions. The spherical volume equivalent diameter $\left(D_{v}\right)$ of the synthetic colloids obtained with sp-ICP-MS is given in Table 4. The size distributions obtained with spICP-MS are number-weighted. The $D_{v}$ of Fh, Gt-NOM and Gt-As differs slightly, but significantly in the order Fh (median $D_{v} 48 \mathrm{~nm}$ ) < Gt-NOM (median $D_{v} 52 \mathrm{~nm}$ ) < Gt-As (median $D_{v} 54 \mathrm{~nm}$ ) (Table S4). The HFO samples could not be analyzed by sp-ICP-MS because no appropriate dilution factor could be determined with the range finding test: with increasing dilution factors the number of detected particles decreased in a non-linear manner, indicating the presence of very small nanoparticles and/or large amounts of ionic Fe. The combination of particle size and mass recoveries by sp-ICP-MS therefore indicates following size ranking for the different colloid samples: HFO colloids $<$ Fh $<$ Gt-NOM $<$ Gt-As, which agrees with the ranking based on FIFFF measurements.

\subsection{Soil solutions}

The organic matter rich and low pH soil (SS-Rhy) has about 10 times lower Fe concentration in the soil solution compared to the mineral soil (SS-Sid). Dialysis indicates that about $12 \%$ of Fe is truly dissolved in the acid organic soil, whereas the mineral soil contains negligible amounts $(<1 \%)$ of truly dissolved Fe. Membrane filtration and centrifugation indicate that large particles are present, especially in the mineral soil where Fe is predominantly present in the fraction $>200 \mathrm{~nm}$. About $70 \%$ of Fe is smaller than $100 \mathrm{~nm}$ in the organic rich, acid soil and only about $6 \%$ in the mineral soil based on centrifugation. Colloidal Fe is detected with 
sp-ICP-MS, but surprisingly the Fe colloids in both soil solutions have a similar size distribution (Figure 5). Again, the mass fraction of Fe detected as particles with sp-ICP-MS is relatively low, $45 \pm 11$ for SS-Rhy and $31 \pm 3 \%$ for SS-Sid. The FIFFF measurements show that both soil solutions contain a large amount of DOC indicated by the large UV peak at small size $(<3 \mathrm{~nm})$. The organic matter rich, acidic soil contains Fe-organic matter complexes and small Fe rich particles (median $D_{h} \sim 15 \mathrm{~nm}$ ) in the soil solution, whereas the $\mathrm{pH}$ neutral, mineral soil predominantly contains larger particles $\left(D_{h} \sim 30-200 \mathrm{~nm}\right)$ in the soil solution (Figure 3).

\section{Discussion}

This study was set up to evaluate the potential of sp-ICP-MS/MS and FIFFF-UV-ICP-MS to determine the size distribution of FeOx colloids and to compare these with more conventional fractionation or sizing methods. In this section, the size distributions of the various methods are compared, taking into account the differences in the type of size determined $\left(D_{v}\right.$ versus $\left.D_{h}\right)$ and in the type of distribution, i.e. mass- or number-weighed. The relative large detection limits of sp-ICP-MS, DLS and NTA did not allow comparison of the size distributions of the small particles, for that reason, this discussion starts with the larger Gt particles for which all methods yielded acceptable fractions on which the determination of size was possible.

\subsection{Goethite colloids.}

The TEM analysis shows that the Gt is elongated with an aspect ratio of about 4 . This means that the $D_{h}$ measured with FIFFF is larger than the rod length obtained with TEM and the $D_{v}$ from sp-ICP-MS analysis is in between rod length and width. Table 4 shows that the particle size obtained with the various techniques mostly differ significantly among the methods. To do a proper size comparison among the methods, number-based size distributions from sp- 
ICP-MS, TEM and NTA on the Gt-NOM particles were converted to mass-based distributions by defining size bins and calculating the total particle mass in a size bin by multiplying particle number with mass of an individual particle in each size bin. That conversion used the particle density of the goethite mineral $\left(4 \mathrm{~g} / \mathrm{cm}^{3}[48]\right)$ and was assumed to be constant over the whole size distribution. This particle density might be a reasonable approximation for TEM and spICP-MS because only the Fe core is measured, it is likely less appropriate to apply the same density for NTA that measure sizes including the coating and the hydration water. From the TEM pictures a volume-equivalent particle diameter was calculated for each detected particle using particle length and width assuming rod shape. In sp-ICP-MS, a large fraction of the particles is below the size detection limit. By converting the number-weighted size distribution to a mass-weighed size distribution, a correction for this recovery was made. The conversion between number and mass distributions, the recovery correction in sp-ICP-MS and the calculation of volume-equivalent diameters from TEM pictures require a few assumptions, the approach is more extensively explained in the supplementary material. A mass-weighed distribution is obtained with dialysis, membrane filtration and centrifugation.

Figure 4 and Table 5 compare the mass-based size distribution of the GT-NOM particles among the techniques. The FIFFF results resemble the $D_{h}$ measured with the NTA and DLS and suggest that the hydrodynamic diameters are about two or three times larger than $D_{v}$ measured with TEM and sp-ICP-MS. The FIFFF is not significantly different from DLS, that measures an intensity-weighted distribution, which is similar to mass [49]. The difference between $D_{h}$ (FIFFF, DLS, NTA) and $D_{v}(s p-I C P-M S, T E M)$ cannot be explained by taking particle shape or distribution weighing factor into account. Conversion of the number-based Gt-NOM distribution from sp-ICP-MS to a mass-based distribution only results in slight shift towards 
larger size because a fairly monodisperse distribution was measured. For non-spherical particles one can expect to measure different size because different particle properties are measured (hydrodynamic diameter and particle volume) that, in turn, depend on aspect ratio. For instance, rod shape particles have higher friction coefficient than spheres with the same volume and therefore larger $D_{h}[50]$. For low aspect ratio rod shape particles, like the Gt particles, the difference in volume equivalent spherical diameter and hydrodynamic diameter is very small (supplementary material). Indeed, for rod shape particles with aspect ratio 4, the volume equivalent diameter and hydrodynamic diameter should in theory only differ about $4 \%$ [50]. The relatively large hydrodynamic diameter of NOM coated Fe oxides (compared to the uncoated particles or techniques that measure the Fe core, like TEM) has been observed in other studies and was attributed to slight particle aggregation or change in particle conformation that decreases the diffusion coefficient [17], [18].

Figure 4 and Table 5 show that the sp-ICP-MS data is very consistent with TEM data for which a mass-based volume equivalent diameter was calculated using rod length and width of primary particles. This suggest that with sp-ICP-MS primary particles were measured. All techniques, including the fractionation techniques, indicate a (significantly) larger size for GtAs compared to Gt-NOM which points to increased particle aggregation with As compared to NOM (Table 3 and S4). This size difference is most pronounced for the hydrodynamic diameters, for instance the $D_{h} 50$ or Z-average diameter is consistently $30 \%$ larger for Gt-As compared to Gt-NOM. The size difference between Gt-As and Gt-NOM is much smaller for spICP-MS, the $D_{v} 50$ of Gt-As is less than $5 \%$ larger than the $D_{v} 50$ of Gt-NOM (Table 4).

The agreement of sp-ICP-MS with TEM and the small size difference between the Gt particles with sp-ICP-MS analysis suggest that the extremely high sample dilution with ultrapure water 
(dilution factors of about $2 \times 10^{6}$ ) in sp-ICP-MS analysis might have resulted in particle disaggregation (in the Gt-NOM sample as well as Gt-As). Decreasing ionic strength expands the electrical double layer and increases the particle-particle repulsion, according to the DLVO (Derjaguin, Landau, Vervey, and Overbeek) colloid stability theory. Very little is known about the reversibility of Fe oxide particle aggregation, but it has been shown that Fe oxides can disaggregate due to increase in surface charge, for instance upon addition of humic substances [16]. It is well known that filtration can cause serious artefacts for colloids because of pore clogging, aggregation with retained particles or sorption on the membranes [11]. This study confirms that filtration yields a dramatic underestimation of the GT particles in the smallest size fractions: $>90 \%$ of Fe is filtered out with the $0.2 \mu \mathrm{m}$ membrane even though the Gt particles are smaller than this cut-off as established with all other methods. In contrast to membrane filtration, centrifugation tends to only slightly underestimate the particle size distribution compared to FIFFF. As discussed above, the density of the FeOx core was used to calculate centrifugation time and speed but hydrodynamic diameters include the contribution of NOM coating, hydration water and aggregation and therefore will have lower particle density than a single solid Fe particle of that size would have. Dialysis and FIFFF yield both the same result that there is a negligible concentration of truly dissolved Fe in the Gt samples.

\subsection{Hydrous Ferric Oxides.}

The FIFFF-UV-ICP-MS clearly revealed that increasing NOM concentration in the presence of oxidizing $\mathrm{Fe}^{2+}$ decreased the size of the HFO colloids (Figure 2). At high $\mathrm{C} / \mathrm{Fe}$, Fe-NOM complexes are formed which inhibits Fe(III) hydrolysis and polymerization, but when the complexation capacity of NOM is exceeded FeOx are formed for which NOM acts as stabilizing agent. 
We recall that for the highest NOM dose (HFO $110 \mathrm{C} / \mathrm{Fe}$ ), speciation calculation indicated that all Fe can be complexed with organic matter. That is observed in the fractogram of HFO 110 $\mathrm{C} /$ Fe because the main Fe and NOM (UV) peak overlap at small size $(<3 n m)$ (Figure 2). When the $\mathrm{C} / \mathrm{Fe}$ ratio decreases, the fractograms show that organo-mineral Fe colloids are formed with increasing size and decreasing amount of NOM associated with the particles, because less NOM is available to inhibit polymerization of Fe.

In HFO $45 \mathrm{C} / \mathrm{Fe}$ a combination of mononuclear complexes and small organo-mineral FeOx coexist because the Fe and NOM peak are partly shifted (Figure S5). There are no Fe-NOM complexes identified in the two samples with lowest organic matter content, HFO 5 and 2.5 C/Fe (Figure 2 and S5). Instead these samples contain polymerized Fe of 10 and $25 \mathrm{~nm}$ size associated with NOM, as indicated by the small UV peak coinciding with the Fe peak. In the sample with the lowest NOM concentration, HFO colloids are relatively large and polydisperse suggesting that organic matter content becomes limiting to suppress further polymerization. Indeed, further decreasing the OM content resulted in large aggregates that settled out solution, as observed in previous studies [17], [18]. The domination of mononuclear Fe-OM complexes at $\mathrm{pH} 6$ at high molar $\mathrm{C} / \mathrm{Fe}$ ratio and increased polymerization at higher $\mathrm{Fe}$ concentrations has been observed with spectroscopic studies [4], [5], [51]. These results are in agreement with the recent study of Guenet et al. [51] who discovered using SAXS/SANS and XAS analysis that the increase in particle size with decreasing organic matter content is due to aggregation of intermediate sized nano-oxide particles .

The small particle size of the HFO particles explains why sp-ICP-MS analysis did not detect particles. The DLS found increasing scattering intensity in the HFO samples as the NOM concentrations decrease, suggesting increased colloid size at decreasing molar C/Fe. 
However, no hydrodynamic diameter could be calculated with DLS due to insufficient scattering intensities, except for HFO $2.5 \mathrm{C} /$ Fe that shows a bimodal distribution with particles of about $40 \mathrm{~nm}$ predominantly present, which fits to FIFFF-data to a certain extent. Colloids with $D_{h} \ll<5 n$ m, cannot be reliably measured with NTA because the larger diffusion lengths will move the particles out of focus and the track is lost.

Dialysis confirms the FIFFF measurements that increasing C/Fe ratio yields increasing fractions of very small organic mononuclear Fe colloids in the HFO samples. The $12 \mathrm{kDa}$ size cut-off in dialysis correspond approximately to a particle size off $3 \mathrm{~nm}$ [46]. This so called truly dissolved fraction contains ionic Fe and Fe complexes with small organic matter species, like fulvic acids. In FIFFF the size range 0-3 nm will contain Fe organic matter complexes, not ionic Fe because ions are supposed to pass the membrane $(1 \mathrm{kDa})$ during focusing. However, ionic Fe will be low in our samples anyway because Fe(III) has very low solubility in neutral, oxic conditions. The higher fraction of Fe complexed with organic matter predicted with FIFFF compared to dialysis (e.g. 64\% with FIFFF and 20\% with dialysis for HFO $110 \mathrm{C} / \mathrm{Fe}$ ) might be explained by the carrier ionic strength that governs size accuracy in FIFFF [27]. The relatively low carrier ionic strength in this study had ample effect on elution time of the coated FeOx particles (Figure S2) but pre-elution of SRNOM was observed under these conditions [27]. Early elution results in underestimation of size in FIFFF when retention time is converted to particle size.

The FIFFF determined particle size of the HFO was below the smallest size fraction of membrane filtration and centrifugation, except for the lowest $\mathrm{C} / \mathrm{Fe}$ ratio, therefore these techniques could not differentiate in particle size between HFO samples. In contrast to the biased results with the Gt colloids, here filtration correctly showed that Fe is predominantly 
present in the fraction $<25 \mathrm{~nm}$, except for the largest HFO particles for which filtration artefacts did occur, underestimating the presence of small particles.

\subsection{Ferrihydrite colloids}

The Fh particles have significantly smaller median $D_{h}$ measured with FIFFF compared to both DLS and NTA (Table 4). The wide particle size distribution of Fh might explain the bias towards larger size for NTA and DLS compared to FIFFF because both NTA and DLS rely on light scattering which has a sixth power relation with particle diameter and, therefore, gives more weight to larger particles. Indeed, TEM pictures show very disperse distribution in sizes with Fe particles of about $10 \mathrm{~nm}$ but also $>100 \mathrm{~nm}$. The three measurements of $D_{h}$ are considerably larger than the size of the primary Fh particles at synthesis $\mathrm{pH}$ without As [34], [35], indicating that the addition of As to stabilize the Fh particles at $\mathrm{pH} 6$ resulted in charge reversal (Table 1) but that the primary Fh particles are agglomerated or aggregated to larger size. The $D_{v}$ obtained with sp-ICP-MS is considerably smaller than the $D_{h}$ given that the $D_{v} 90$ is only $59 \mathrm{~nm}$ and that the Fe mass recovery with sp-ICP-MS analysis is only $9 \%$. As concluded from the Gt samples, we hypothesize that the high dilution in ultrapure water (for Fh dilution factors of about $5 \times 10^{5}$ ) in $\mathrm{sp}-\mathrm{ICP}-\mathrm{MS}$ analysis resulted in increased particle-particle repulsion and disaggregation into particles that are below the size detection limit in sp-ICP-MS (about 42 $\mathrm{nm}$ for Fh). Again, the size distribution obtained with centrifugation is closer to the distribution from FIFFF than sp-ICP-MS measurements and membrane filtration underestimates the smallest colloidal fraction.

\subsection{Soil solutions}


The soil solutions have contrasting properties (Table 2). The high DOC content and low $\mathrm{pH}$ of the Rhytalog soil promotes Fe-NOM complexation and the high soil solution $\mathrm{Ca}^{2+}$ concentration flocculates organo-mineral Fe colloids. The SS-Sid sample on the other hand has low DOC, relatively high $\mathrm{pH}$ and low $\mathrm{Ca}^{2+}$, factors that promote presence of FeOx colloids. In the soil solutions, both FIFFF and sp-ICP-MS detected colloidal Fe particles. As anticipated from soil characteristics, FIFFF analysis shows small $(<50 \mathrm{~nm})$ Fe-organic matter associations in the acid, organic rich soil and larger $(30-150 \mathrm{~nm})$ Fe rich particles in the $\mathrm{pH}$ neutral mineral soil. These results contrast single-particle ICP-MS data that show very similar FeOx size distribution for both soil solutions (Figure 5). Multi-element analysis on the FIFFF fractogram (not shown) showed co-elution of Al together with Fe at molar Al/Fe ratio of 4 in SS-Sid, which might be an indication that Fe is present as clay particles or as Fe oxyhydroxide coating on clay particles. Colloids with size similar to the particles found in SS-Sid were identified as clay minerals in the study by Regelink et al. [25]. If clay particles or FeOx coating on clay particles would be present, particle size will be underestimated with single-particle ICP-MS analysis because an assumption is made on particle density and stoichiometry to convert the mass of analyte to the particle size. In clay particles, Fe is only a small fraction [52] resulting in underestimation of the full clay particle size that mainly consist of other elements. In addition, clay particles have lower density hence larger size for the same particles mass compared to FeOx [48].

Membrane filtration dramatically underestimates colloidal Fe in SS-Sid, the filter might have been clogged due to some large particles in the soil solution. The SS-Sid is also the only sample where FIFFF clearly measures a smaller size distribution compared to centrifugation. It is possible that in the FIFFF analysis larger particles are not included (because they are retained 
in the nebulizer or because ionization of large particles might have been incomplete) shifting the size distribution towards smaller size, especially in a mass-based distribution, in comparison with centrifugation, where $\mathrm{Fe}$ is determined after aqua regia digestion. In addition, centrifugation might have affected colloidal stability or resulted in drag of small particles when large settling particles are present. Gimbert et al. [11] recentrifuged the pellet obtained after centrifugation multiple times to ensure that all particles smaller than a certain fraction are recovered in the supernatant and found good agreement between centrifugation and Centrifugal Field Flow Fractionation for soil suspensions.

\section{Conclusion}

We explored the ability of two innovative techniques in nanoparticle analysis, Flow Field Flow Fractionation (FIFFF) and single-particle ICP-MS, to size organo-mineral FeOx colloids. The FIFFF-UV-ICP-MS and sp-ICP-MS/MS methods were optimized to obtain high recovery and low size detection limits and were tested on a set of simplified synthetic organo-mineral FeOx colloids with experimentally varied properties.

With FIFFF a wide particle size range could be measured with good recovery (> $70 \%)$. In particular, the method allowed for high size resolution at small particle size and distinguished between Fe-OM complexes $(<5 \mathrm{~nm})$ and small organo-mineral Fe-Ox colloids. In contrast, single-particle ICP-MS analysis using triple quadrupole technology (ICP-MS/MS), resulted in a size detection limit for FeOx of about $32-47 \mathrm{~nm}$ and was therefore not capable to obtain the FeOx particle size in the low nanometer range. The potential of a Sector Field ICP-MS, which reduces polyatomic interferences with higher mass resolution, should be explored to potentially further decrease the size detection limit for FeOx colloids in single-particle ICP-MS analysis. 
In the soil solution obtained with centrifugation of an acid peat, FIFFF-UV-ICP-MS analysis showed Fe-OM complexes and small organo-mineral Fe colloids $(<30 \mathrm{~nm})$, whereas that of a mineral pH neutral soil mainly contained larger $(30-200 \mathrm{~nm})$ Fe rich particles. In sp-ICP-MS analysis, the uncertainty of Fe speciation in complex environmental samples impedes the calculation of particle size because an assumption on Fe mass fraction and particle density has to be made. The assumption might lead to erroneous size results because Fe can be present as FeOx colloids, as FeOx coating or in clay particles.

FIFFF and sp-ICP-MS results cover different information on particle dimensions. The sp-ICPMS measured the size of the Fe core of primary particles similarly to TEM analysis, whereas FIFFF measured the hydrodynamic diameter of aggregated particles. However, given the ability of FIFFF to detect FeOx colloids down to the low nanometer range, which is of particular environmental interest, FIFFF seems to be more relevant to study organo-mineral FeOx in the environment.

\section{Appendix A: Supplementary material}

Acknowledgements: The authors wish to thank Pieter-Jan De Temmerman for assistance in TEM and NTA measurements. The research was funded by KULeuven. 


\section{References}

[1] A. Voegelin, R. Kaegi, J. Frommer, D. Vantelon, and S. J. Hug, "Effect of phosphate, silicate, and Ca on $\mathrm{Fe}(\mathrm{III})$-precipitates formed in aerated $\mathrm{Fe}(\mathrm{II})$ - and As(III)-containing water studied by X-ray absorption spectroscopy," Geochim. Cosmochim. Acta, vol. 74, no. 1, pp. 164-186, 2010.

[2] U. Schwertmann and R. M. Cornell, Iron Oxides in the Laboratory. 1992.

[3] Cornell, R. M.; Schwertmann, U., The Iron Oxides., 2nd ed. 2003.

[4] C. Sjöstedt, I. Persson, D. Hesterberg, D. B. Kleja, H. Borg, and J. P. Gustafsson, "Iron speciation in softwater lakes and soils as determined by EXAFS spectroscopy and geochemical modelling," Geochim. Cosmochim. Acta, vol. 105, pp. 172-186, 2013.

[5] T. Karlsson and P. Persson, "Complexes with aquatic organic matter suppress hydrolysis and precipitation of Fe(III)," Chem. Geol., vol. 322-323, pp. 19-27, 2012.

[6] S. Lofts, E. Tipping, and J. Hamilton-Taylor, "The chemical speciation of Fe(III) in freshwaters," Aquat. Geochemistry, vol. 14, no. 4, pp. 337-358, 2008.

[7] M. Hassellöv and F. von der Kammer, "Iron oxides as geochemical nanovectors for metal transport in soilriver systems," Elements, vol. 4, no. 6, pp. 401-406, 2008.

[8] S. A. Bradford, J. Simunek, M. Bettahar, M. T. Van Genuchten, and S. R. Yates, "Significance of straining in colloid deposition: Evidence and implications," Water Resour. Res., vol. 42, no. 12, pp. 1-16, 2006.

[9] J. Gimpel, H. Zhang, W. Davison, and A. C. Edwards, "In situ trace metal speciation in lake surface waters using DGT, dialysis, and filtration," Environ. Sci. Technol., vol. 37, no. 1, pp. 138-146, 2003.

[10] S. Baken, C. Moens, B. van der Grift, and E. Smolders, "Phosphate binding by natural iron-rich colloids in streams," Water Res., vol. 98, pp. 326-333, 2016.

[11] L. J. Gimbert, P. M. Haygarth, R. Beckett, and P. J. Worsfold, "Comparison of centrifugation and filtration techniques for the size fractionation of colloidal material in soil suspensions using sedimentation fieldflow fractionation," Environ. Sci. Technol., vol. 39, no. 6, pp. 1731-1735, 2005.

[12] C. Pelekani, G. Newcombe, V. L. Snoeyink, C. Hepplewhite, S. Assemi, and R. Beckett, "Characterization of natural organic matter using high performance size exclusion chromatography," Environ. Sci. Technol., vol. 33, no. 16, pp. 2807-2813, 1999.

[13] E. Neubauer, F. V.D. Kammer, and T. Hofmann, "Using FLOWFFF and HPSEC to determine trace metalcolloid associations in wetland runoff," Water Res., vol. 47, no. 8, pp. 2757-2769, 2013.

[14] B. Meermann and F. Laborda, "Analysis of nanomaterials by field-flow fractionation and single particle ICP-MS," J. Anal. At. Spectrom., vol. 30, no. 6, pp. 1226-1228, 2015.

[15] J. C. Giddings, "Field-Flow-Fractionation -Analysis of macromolecular, colloidal, and particulate materials," Science (80-. )., vol. 260, no. 5113, pp. 1456-1465, 1993.

[16] M. Baalousha, "Aggregation and disaggregation of iron oxide nanoparticles: Influence of particle concentration, pH and natural organic matter," Sci. Total Environ., vol. 407, no. 6, pp. 2093-2101, 2009.

[17] M. ; Baalousha, A. ; Manciulea, S. ; Cumberland, K. ; Kendall, and J. R. Lead, "Aggregation and Surface Properties of Iron Oxide Nanoparticles," Environ. Toxicol. Chem., vol. 27, no. 9, 2008.

[18] L. Chekli, S. Phuntsho, M. Roy, and H. K. Shon, "Characterisation of Fe-oxide nanoparticles coated with 
humic acid and Suwannee River natural organic matter," Sci. Total Environ., vol. 461-462, pp. 19-27, 2013.

[19] B. Stolpe, L. Guo, A. M. Shiller, and M. Hassellöv, "Size and composition of colloidal organic matter and trace elements in the Mississippi River, Pearl River and the northern Gulf of Mexico, as characterized by flow field-flow fractionation," Mar. Chem., vol. 118, no. 3-4, pp. 119-128, 2010.

[20] B. Lyvén, M. Hassellöv, D. R. Turner, C. Haraldsson, and K. Andersson, "Competition between iron- and carbon-based colloidal carriers for trace metals in a freshwater assessed using flow field-flow fractionation coupled to ICPMS," Geochim. Cosmochim. Acta, vol. 67, no. 20, pp. 3791-3802, 2003.

[21] S. Baken, I. C. Regelink, R. N. J. Comans, E. Smolders, and G. F. Koopmans, "Iron-rich colloids as carriers of phosphorus in streams: A field-flow fractionation study.," Water Res., vol. 99, pp. 83-90, 2016.

[22] N. Gottselig, R. Bol, V. Nischwitz, H. Vereecken, W. Amelung, and E. Klumpp, "Distribution of PhosphorusContaining Fine Colloids and Nanoparticles in Stream Water of a Forest Catchment," Vadose Zo. J., vol. 13, no. 7, p. 0, 2014.

[23] T. Saito, T. Hamamoto, T. Mizuno, T. Iwatsuki, and S. Tanaka, "Comparative study of granitic and sedimentary groundwater colloids by flow-field flow fractionation coupled with ICP-MS," J. Anal. At. Spectrom., vol. 30, no. 6, pp. 1229-1236, 2015.

[24] D. J. Lapworth, B. Stolpe, P. J. Williams, D. C. Gooddy, and J. R. Lead, "Characterization of suboxic groundwater colloids using a multi-method approach," Environ. Sci. Technol., vol. 47, no. 6, pp. 25542561, 2013.

[25] I. C. Regelink, A. Voegelin, L. Weng, G. F. Koopmans, and R. N. J. Comans, "Characterization of Colloidal Fe from Soils Using Field-Flow Fractionation and Fe K - Edge X - ray Absorption Spectroscopy," Environ. Sci. Technol., vol. 48, pp. 4307-4316, 2014.

[26] A. Missong et al., "Phosphorus in water dispersible-colloids of forest soil profiles," Plant Soil, vol. 427, no. 1-2, pp. 71-86, 2018.

[27] E. Neubauer, F. V.d. Kammer, and T. Hofmann, "Influence of carrier solution ionic strength and injected sample load on retention and recovery of natural nanoparticles using Flow Field-Flow Fractionation," J. Chromatogr. A, vol. 1218, no. 38, pp. 6763-6773, 2011.

[28] M. Baalousha, F. V. D. Kammer, M. Motelica-Heino, and P. Le Coustumer, "Natural sample fractionation by FIFFF-MALLS-TEM: Sample stabilization, preparation, pre-concentration and fractionation," J. Chromatogr. A, vol. 1093, no. 1-2, pp. 156-166, 2005.

[29] F. Laborda, E. Bolea, and J. Jiménez-Lamana, "Single particle inductively coupled plasma mass spectrometry: A powerful tool for nanoanalysis," Anal. Chem., vol. 86, no. 5, pp. 2270-2278, 2014.

[30] C. Degueldre and P. Y. Favarger, "Colloid analysis by single particle inductively coupled plasma-mass spectroscopy: A feasibility study," Colloids Surfaces A Physicochem. Eng. Asp., vol. 217, no. 1-3, pp. 137142, 2003.

[31] M. A. Gomez-Gonzalez, E. Bolea, P. A. O’Day, J. Garcia-Guinea, F. Garrido, and F. Laborda, “Combining single-particle inductively coupled plasma mass spectrometry and X-ray absorption spectroscopy to evaluate the release of colloidal arsenic from environmental samples," Anal. Bioanal. Chem., vol. 408, no. 19, pp. 5125-5135, 2016.

[32] S. Lee, X. Bi, R. B. Reed, J. F. Ranville, P. Herckes, and P. Westerhoff, "Nanoparticle size detection limits by single particle ICP-MS for 40 elements," Environ. Sci. Technol., vol. 48, no. 17, pp. 10291-10300, 2014.

[33] J. Bollyn et al., "Polyphosphates and Fulvates Enhance Environmental Stability of PO4-Bearing Colloidal 
Iron Oxyhydroxides," J. Agric. Food Chem., vol. 64, no. 45, pp. 8465-8473, 2016.

[34] D. J. Burleson and R. L. Penn, "Two-step growth of goethite from ferrihydrite," Langmuir, vol. 22, no. 1, pp. 402-409, 2006.

[35] J. Bollyn, J. Faes, A. Fritzsche, and E. Smolders, "Colloidal-Bound Polyphosphates and Organic Phosphates Are Bioavailable: A Nutrient Solution Study," J. Agric. Food Chem., vol. 65, no. 32, pp. 6762-6770, 2017.

[36] S. Baken, S. Nawara, C. Van Moorleghem, and E. Smolders, "Iron colloids reduce the bioavailability of phosphorus to the green alga Raphidocelis subcapitata," Water Res., vol. 59, pp. 198-206, 2014.

[37] R. H. Loeppert and D. L. Suarez, "Carbonate and Gypsum," in Methods of Soil Analysis Part 3-Chemical Methods, 5.3., D. L. Sparks, A. L. Page, P. A. Helmke, and R. H. Loeppert, Eds. SSSA, ASA, Madison, 1996.

[38] U. Schwertmann, "Differenzierung der Eisenoxide des Bodens durch Extraktion mit AmmoniumoxalatLösung," Pflanzenernähr. Düng. Bodenk., vol. 105, no. 3, pp. 194-202, 1964.

[39] J. L. Weishaar, M. S. Fram, R. Fujii, and K. Mopper, "Evaluation of Specific Ultraviolet Absorbance as an Indicator of the Chemical Composition and Reactivity of Dissolved Organic Carbon," Environ. Sci. Technol., vol. 37, pp. 4702-4708, 2003.

[40] B. A. Poulin, J. N. Ryan, and G. R. Aiken, "The effects of iron on optical properties of dissolved organic matter.," Environ. Sci. Technol. Technol., vol. 48, no. 17, pp. 10098-10106, 2014.

[41] c. Stephan and A. Hineman, "Iron nanoparticles by SP-ICP-MS: overcoming spectral interferences using universal cell technology. Application Brief ICP-Mass Spectrometry," Shelton, CT, USA, 2014.

[42] H. E. Pace, N. J. Rogers, C. Jarolimek, V. A. Coleman, C. P. Higgins, and J. F. Ranville, “Determining transport efficiency for the purpose of counting and sizing nanoparticles via single particle inductively coupled plasma mass spectrometry," Anal. Chem., vol. 83, no. 24, pp. 9361-9369, 2011.

[43] R. Peters et al., "Single particle ICP-MS combined with a data evaluation tool as a routine technique for the analysis of nanoparticles in complex matrices," J. Anal. At. Spectrom., vol. 30, no. 6, pp. 1274-1285, 2015.

[44] J. Tuoriniemi, G. Cornelis, and M. Hassellöv, "Size discrimination and detection capabilities of singleparticle ICPMS for environmental analysis of silver nanoparticles," Anal. Chem., vol. 84, no. 9, pp. 39653972, 2012.

[45] X. Wang, F. Liu, W. Tan, W. Li, X. Feng, and D. L. Sparks, "Characteristics of phosphate adsorptiondesorption onto ferrihydrite: Comparison with well-crystalline fe (Hydr)oxides," Soil Sci., vol. 178, no. 1, pp. 1-11, 2013.

[46] H. P. Erickson, "Size and shape of protein molecules at the nanometer level determined by sedimentation, gel filtration, and electron microscopy," Biol. Proced. Online, vol. 11, no. 1, pp. 32-51, 2009.

[47] L. Balcaen, E. Bolea-Fernandez, M. Resano, and F. Vanhaecke, "Inductively coupled plasma - Tandem mass spectrometry (ICP-MS/MS): A powerful and universal tool for the interference-free determination of (ultra)trace elements - A tutorial review," Anal. Chim. Acta, vol. 894, pp. 7-19, 2015.

[48] H. Cresswell and G. Hamilton, "Bulk density and pore space relations" in: Soil physical measurement and interpretation for land evaluation. Australian Soil and Land Survey Handbook. Melbourne: CSIRO, 2002.

[49] J. P. F. G. Helsper et al., "Physicochemical characterization of titanium dioxide pigments using various techniques for size determination and asymmetric flow field flow fractionation hyphenated with inductively coupled plasma mass spectrometry," Anal. Bioanal. Chem., vol. 408, no. 24, pp. 6679-6691, 2016. 
[50] Jennings B R and Parslow K, "The equivalent spherical diameter," Proc. R. Soc. A., Math. Phys. Sci., vol. 419, p. 137, 1988.

[51] H. Guénet et al., "Characterization of iron-organic matter nano-aggregate networks through a combination of SAXS/SANS and XAS analyses: impact on As binding," Environ. Sci. Nano, vol. 4, no. 4, pp. 938-954, 2017.

[52] H. Van Olphen and J. J. Fripiat, Data Handbook for Clay Materials and Other Non-metallic Minerals, 1st ed. 1979. 
Table 1 Characteristics of synthetic Fe oxyhydroxide colloids, elemental concentrations of arsenic (As) and iron (Fe) refer to measured total concentrations. For the HFO samples, the $\mathrm{C}$ content was varied, the $\mathrm{C} / \mathrm{Fe}$ ratio represent the molar $\mathrm{C} / \mathrm{Fe}$ ratio in test solutions.

\begin{tabular}{ccccccc}
\hline Sample reference & mineralogy & pH & added DOC & As & Fe & Zetapotential \\
& & & $m g L^{-1}$ & $m g L^{-1}$ & $m g L^{-1}$ & $\mathrm{mV}$ \\
\hline \hline Gt-NOM & Goethite & 6.4 & 12.5 & & 54 & -32 \\
Fh & Goethite & 6.5 & & 11.2 & 47 & -39 \\
HFO $110 \mathrm{C} / \mathrm{Fe}$ & Hydrous Ferric oxide & 6.0 & 120 & & 5.0 & n.m. \\
HFO $45 \mathrm{C} / \mathrm{Fe}$ & Hydrous Ferric oxide & 6.3 & 50 & & 4.9 & n.m. \\
HFO $5 \mathrm{C} / \mathrm{Fe}$ & Hydrous Ferric oxide & 6.1 & 5 & & 4.7 & n.m. \\
HFO 2.5 C/Fe & Hydrous Ferric oxide & 6.1 & 2.5 & & 4.6 & n.m. \\
\hline
\end{tabular}


Table 2 Selected soil properties and soil solution (SS) composition of the two soils. The soil solutions are obtained with centrifugation

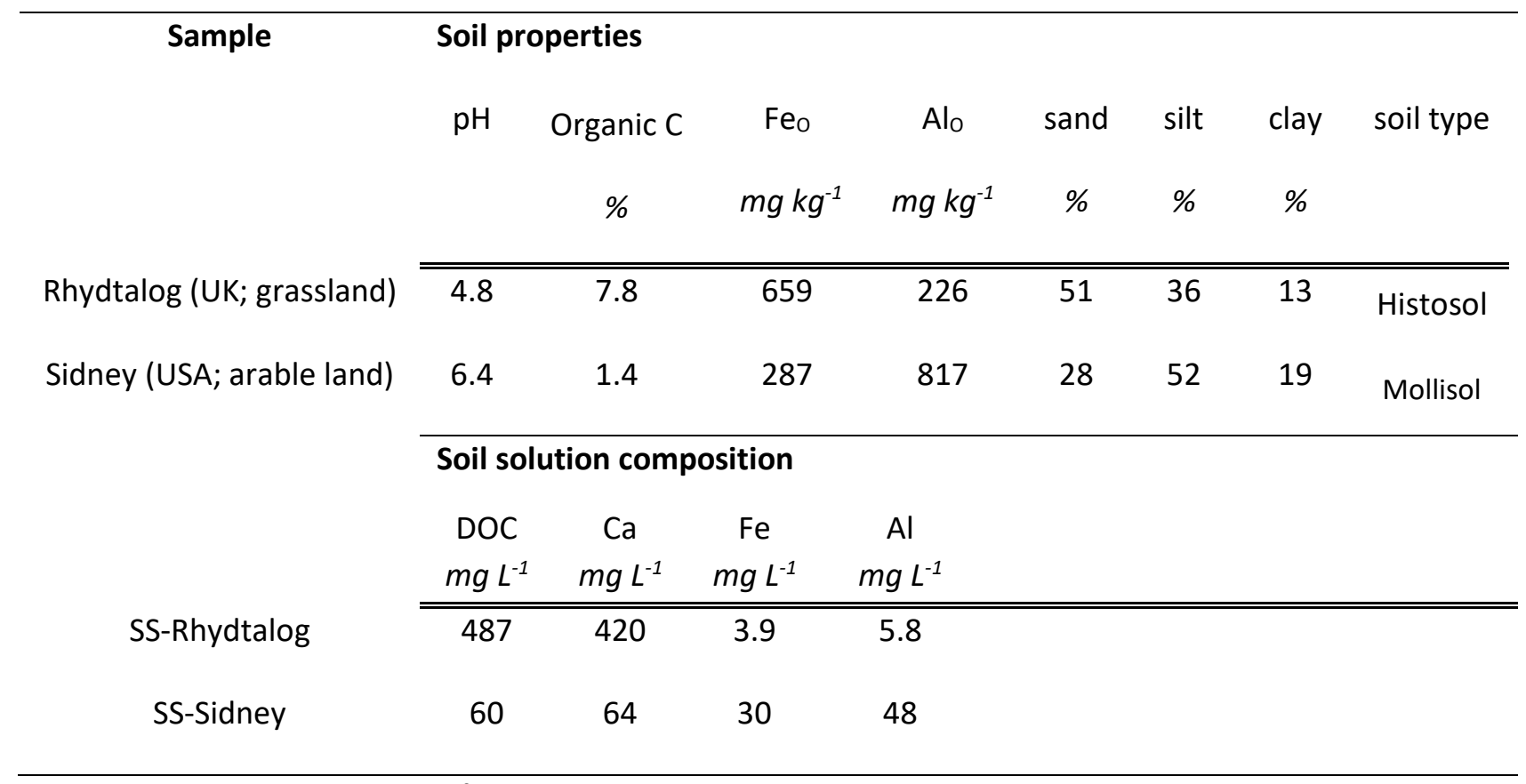

Sand, silt, clay according to USDA classification; $\mathrm{Fe}_{\mathrm{o}}$ and $\mathrm{Al}_{\mathrm{O}}$ : oxalate extractable Fe and Al; DOC Dissolved organic carbon 
Table 3 Fractionation of all samples with dialysis, membrane filtration (MF), centrifugation (CF) and Field Flow Fractionation (FIFFF) given as mean \pm standard deviation.

\begin{tabular}{|c|c|c|c|c|c|c|c|}
\hline \multirow[t]{2}{*}{ sample } & \multirow[t]{2}{*}{ method } & \multirow{2}{*}{$\begin{array}{l}\text { mass fraction (\%) } \\
<3 \mathrm{~nm}\end{array}$} & \multirow[t]{2}{*}{ method } & \multicolumn{4}{|c|}{ mass fraction (\%) } \\
\hline & & & & $0-25 \mathrm{~nm}$ & $25-100 \mathrm{~nm}$ & $100-200 \mathrm{~nm}$ & $200-450 \mathrm{~nm}$ \\
\hline \multirow{3}{*}{ HFO $110 \mathrm{C} / \mathrm{Fe}$} & \multirow{2}{*}{ dialysis } & \multirow{2}{*}{$20 \pm 2$} & MF & $83 \pm 2$ & $17 \pm 2$ & & \\
\hline & & & CF & $95 \pm 4$ & $1 \pm 4$ & & $8 \pm 5$ \\
\hline & FIFFF & $64 \pm 0.1$ & FIFFF & $80 \pm 0.5$ & & & \\
\hline \multirow{3}{*}{$\mathrm{HFO} 45 \mathrm{C} / \mathrm{Fe}$} & \multirow{2}{*}{ dialysis } & \multirow{2}{*}{$9 \pm 4$} & MF & $89 \pm 8$ & $6 \pm 2$ & & $4 \pm 1$ \\
\hline & & & CF & $93 \pm 2$ & $1 \pm 2$ & $4 \pm 1$ & \\
\hline & FIFFF & $21 \pm<0.1$ & FIFFF & $85 \pm 2$ & & & \\
\hline \multirow{3}{*}{$\mathrm{HFO} 5 \mathrm{C} / \mathrm{Fe}$} & \multirow{2}{*}{ dialysis } & \multirow{2}{*}{$2 \pm 1$} & MF & $74 \pm 17$ & $2 \pm 2$ & $21 \pm 2$ & \\
\hline & & & CF & $90 \pm 4$ & $3 \pm 0$ & $1 \pm 2$ & \\
\hline & FIFFF & $1 \pm 0.1$ & FIFFF & $78 \pm 0.5$ & & & \\
\hline \multirow{3}{*}{ HFO $2.5 \mathrm{C} / \mathrm{Fe}$} & \multirow{2}{*}{ dialysis } & \multirow{2}{*}{$1 \pm 0.2$} & MF & $13 \pm 7$ & $23 \pm 3$ & $59 \pm 4$ & $2 \pm 4$ \\
\hline & & & CF & $69 \pm 3$ & $15 \pm 5$ & $3 \pm 2$ & $1 \pm 4$ \\
\hline & FIFFF & $0.2 \pm<0.1$ & FIFFF & $40 \pm 11$ & $55 \pm 4$ & $1 \pm 0.4$ & \\
\hline \multirow{3}{*}{$\mathrm{Fh}$} & \multirow{2}{*}{ dialysis } & \multirow{2}{*}{$4 \pm 1$} & MF & & $32 \pm 10$ & $28 \pm 11$ & $42 \pm 3$ \\
\hline & & & $\mathrm{CF}$ & $41 \pm 2$ & $57 \pm 1$ & $3 \pm 2$ & \\
\hline & FIFFF & $0.2 \pm 0.2$ & FIFFF & $5 \pm 1$ & $55 \pm 12$ & $23 \pm 4$ & $2 \pm 0.5$ \\
\hline \multirow{3}{*}{ Gt-NOM } & \multirow{2}{*}{ dialysis } & \multirow{2}{*}{$3 \pm 1$} & MF & & $13 \pm 6$ & & $90 \pm 1$ \\
\hline & & & CF & $16 \pm 0.1$ & $68 \pm 3$ & $14 \pm 2$ & $3 \pm 1$ \\
\hline & FIFFF & $0.1 \pm<0.1$ & FIFFF & $0.4 \pm 0.1$ & $42 \pm 8$ & $38 \pm 7$ & $3 \pm 3$ \\
\hline \multirow{3}{*}{ Gt-As } & \multirow{2}{*}{ dialysis } & \multirow{2}{*}{$1 \pm 1$} & MF & $1 \pm 1$ & $6 \pm 1$ & & $95 \pm 2$ \\
\hline & & & CF & $2 \pm 0.4$ & $63 \pm 4$ & $26 \pm 1$ & $15 \pm 1$ \\
\hline & FIFFF & $0.3 \pm 0.1$ & FIFFF & $0.7 \pm 0.1$ & $22 \pm 7$ & $42 \pm 6$ & $22 \pm 5$ \\
\hline \multirow[t]{3}{*}{ SS-Rhy } & \multirow{2}{*}{ dialysis } & \multirow{2}{*}{$12 \pm 3$} & MF & $14 \pm 2$ & $15 \pm 1$ & & $38 \pm 4$ \\
\hline & & & $\mathrm{CF}$ & $32 \pm 1$ & $37 \pm 1$ & $21 \pm 1$ & $10 \pm 0.4$ \\
\hline & FIFFF & $10 \pm 3$ & FIFFF & $52 \pm 6$ & $25 \pm 5$ & $8 \pm 4$ & \\
\hline \multirow[t]{3}{*}{ SS-Sid } & \multirow{2}{*}{ dialysis } & & MF & $0.9 \pm 1$ & & $8 \pm 11$ & $4 \pm 2$ \\
\hline & & $0.3 \pm 0.5$ & $\mathrm{CF}$ & $1 \pm 0.2$ & $5 \pm 0.3$ & $10 \pm 1$ & $84 \pm 7$ \\
\hline & FIFFF & $1 \pm 1$ & FIFFF & $4 \pm 1$ & $47 \pm 0.6$ & $18 \pm 0.5$ & \\
\hline
\end{tabular}

Empty fields correspond with concentrations below the ICP-MS detection limit; The 10-12 kDa dialysis size fraction approximately corresponds to a particle size of $3 \mathrm{~nm}$. 
Table 4 Overview of equivalent spherical diameters for all synthetic samples; hydrodynamic diameter $D_{h}$ measured with FIFFF, DLS and NTA and spherical volume equivalent diameter $D_{v}$ measured with sp-ICP-MS. The errors on the $D_{h}$ and $D_{v}$ values are standard deviations on replicate measurements. $<S D L=$ below size detection limit; < QL=below quantification limit; D10-D50-D90 represent respective percentiles of the size distribution. The diameters are compared statistically among the different methods with Tukey HSD test, values that are not statistically different $(\alpha=0.05)$ are indicated with the same letter (small letter between brackets for D10; capitals for D50 and capitals between brackets for D90). For DLS the Z-average diameter was compared with the D50 from the other methods, the Z-average diameter is the harmonic mean of the lognormal size distribution.

\begin{tabular}{|c|c|c|c|c|c|c|c|c|c|c|}
\hline \multirow[b]{2}{*}{ Sample } & \multicolumn{3}{|c|}{ FIFFF } & \multirow{2}{*}{$\begin{array}{c}\text { DLS } \\
\text { Dh } \\
(n m)\end{array}$} & \multicolumn{3}{|c|}{ NTA } & \multicolumn{3}{|c|}{ Sp-ICP-MS } \\
\hline & $\mathrm{Dh}_{\mathrm{h}} 10$ & $\begin{array}{l}\text { Dh50 } \\
(\mathrm{nm})\end{array}$ & $D_{h} 90$ & & Dh10 & $\begin{array}{l}\mathrm{Dh}_{\mathrm{h}} 50 \\
\quad(\mathrm{~nm})\end{array}$ & Dh90 & Dv10 & $\begin{array}{l}\text { Dv50 } \\
(\mathrm{nm})\end{array}$ & Dv90 \\
\hline "HFO $110 \mathrm{C} / \mathrm{Fe}$ & $0.9 \pm<0.1$ & $2 \pm<0.1$ & $4 \quad 4 \pm<0.1$ & $\angle \mathrm{CQL}^{3}$ & & $\angle Q \mathrm{QQ}$ & & & 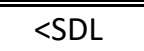 & \\
\hline HFO 45 C/Fe & $2 \pm<0.1$ & $4 \pm 0.1$ & $6 \pm 0.2$ & $<Q^{3}$ & & $<Q L$ & & & $<S D L$ & \\
\hline HFO 5 C/Fe & $6 \pm<0.1$ & $9 \pm<0.1$ & $13 \pm<0.1$ & $<Q L^{3}$ & & $<Q L$ & & & $<S D L$ & \\
\hline HFO $2.5 \mathrm{C} / \mathrm{Fe}$ & $16 \pm 1$ & $27 \pm 3$ & $46 \pm 5$ & 42 and $281^{2}$ & & $<Q L$ & & & $<S D L$ & \\
\hline Fh-As & $\begin{array}{l}31 \pm 1 \\
\text { (b) }\end{array}$ & $\begin{array}{c}67 \pm 4 \\
B\end{array}$ & $\begin{array}{c}136 \pm 14 \\
\text { (A) }\end{array}$ & $\begin{array}{c}102 \pm 30^{1} \\
\mathrm{~A}\end{array}$ & $\begin{array}{l}58 \pm 4 \\
\text { (a) }\end{array}$ & $\begin{array}{c}101 \pm 5 \\
A\end{array}$ & $\begin{array}{c}171 \pm 15 \\
\text { (A) }\end{array}$ & $\begin{array}{l}44 \pm 0.2 \\
\text { (b) }\end{array}$ & $\begin{array}{c}48 \pm 0.5 \\
C\end{array}$ & $\begin{array}{c}59 \pm 1 \\
\text { (B) }\end{array}$ \\
\hline Gt-NOM & $\begin{array}{l}57 \pm 4 \\
\text { (b) }\end{array}$ & $\begin{array}{c}98 \pm 11 \\
\mathrm{~A}\end{array}$ & $\begin{array}{c}162 \pm 8 \\
\text { (A) }\end{array}$ & $\begin{array}{c}84 \pm 12^{1} \\
B\end{array}$ & $\begin{array}{l}51 \pm 1 \\
\text { (a) }\end{array}$ & $\begin{array}{c}77 \pm 1 \\
C\end{array}$ & $\begin{array}{c}132 \pm 4 \\
\text { (B) }\end{array}$ & $\begin{array}{c}48 \pm 0.1 \\
\text { (a) }\end{array}$ & $\begin{array}{c}52 \pm 0.2 \\
D\end{array}$ & $\begin{array}{c}63 \pm 0.4 \\
\text { (C) }\end{array}$ \\
\hline Gt-As & $\begin{array}{c}69 \pm 1 \\
\text { (b) }\end{array}$ & $\begin{array}{c}138 \pm 9 \\
A\end{array}$ & $\begin{array}{c}260 \pm 25 \\
\text { (A) }\end{array}$ & $\begin{array}{c}118 \pm 20^{1} \\
A, B\end{array}$ & $\begin{array}{c}64 \pm 3 \\
\text { (b) }\end{array}$ & $\begin{array}{c}109 \pm 5 \\
B\end{array}$ & $\begin{array}{c}174 \pm 7 \\
\text { (B) }\end{array}$ & $\begin{array}{c}46 \pm 1 \\
\text { (a) }\end{array}$ & $\begin{array}{c}54 \pm 0.3 \\
c\end{array}$ & $\begin{array}{c}72 \pm 0.4 \\
\text { (C) }\end{array}$ \\
\hline
\end{tabular}

${ }_{1}^{1}$ obtained from cumulant analysis and ${ }^{2}$ from multimodal analysis; ${ }^{3} \mathrm{QL}=1003 \times 103$ counts s $^{-1}(9 \sigma$ of continuously measured blank) 
Table 5 Overview of mass-based particle size distributions for Gt-NOM colloids with hydrodynamic diameter $D_{h}$ obtained with FIFFF, DLS and NTA and spherical volume equivalent diameter $D_{v}$ obtained with sp-ICP-MS analysis and TEM. The data corresponds with the size distributions as shown in Figure 5. The errors on the $D_{h}$ and $D_{v}$ values are standard deviations of replicate measurements. D10-D50D90 represent respective percentiles of the size distribution. The diameters are compared among the methods with Tukey HSD test, values that are not statistically different $(\alpha=0.05)$ are indicated with the same letter (small letter between brackets for D10; capitals for D50 and capitals between brackets for D90). For DLS the Z-average diameter was compared with the D50 from the other methods, the Zaverage diameter is the harmonic mean of the lognormal size distribution.

\begin{tabular}{|c|c|c|c|}
\hline \multirow[t]{2}{*}{ Method } & \multicolumn{2}{|c|}{ diameter $(n m)$} & \multirow[b]{2}{*}{ a } \\
\hline & $D_{h} 10$ & $57 \pm 4$ & \\
\hline \multirow[t]{2}{*}{ FIFFF } & $D_{h} 50$ & $98 \pm 8$ & A \\
\hline & $D_{h} 90$ & $163 \pm 11$ & (A) \\
\hline \multirow[t]{2}{*}{ DLS } & $D_{h}$ & $84 \pm 2^{2}$ & $A$ \\
\hline & $\mathrm{D}_{\mathrm{h}} 10$ & $69 \pm 3$ & $\mathrm{~b}$ \\
\hline \multirow[t]{3}{*}{ NTA } & $D_{h} 50$ & $127 \pm 13$ & B \\
\hline & Dh90 & $222 \pm 31$ & (B) \\
\hline & $\mathrm{D}_{\mathrm{v}} 10$ & $<\mathrm{SDL}^{1}$ & \\
\hline \multirow[t]{3}{*}{ sp-ICP-MS } & $D_{v} 50$ & $<\mathrm{SDL}^{1}$ & \\
\hline & $D_{v} 90$ & $63 \pm 1$ & (C) \\
\hline & $\mathrm{D}_{\mathrm{v}} 10$ & 22 & \\
\hline \multirow[t]{2}{*}{ TEM } & $D_{v} 50$ & 40 & \\
\hline & $D_{\vee} 90$ & 56 & \\
\hline
\end{tabular}

${ }^{1}<\mathrm{SDL}=$ below size detection limit of $47 \mathrm{~nm} ;{ }^{2}$ obtained from cumulant analysis 


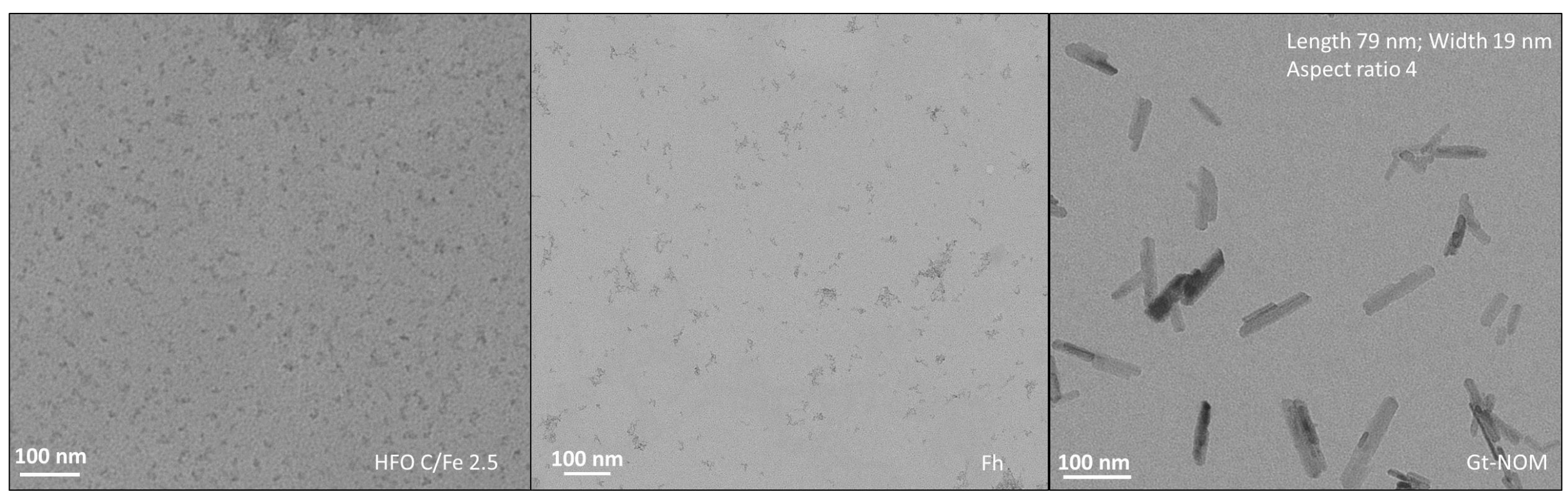

Figure 1 TEM analysis on selected samples; The Hydrous Ferric Oxides (HFO, left) and ferrihydrite (Fh, middle) have irregular shape but Fh contains larger particles than HFO. The goethite (Gt) particles have a defined rod shape with median aspect ratio 4. 

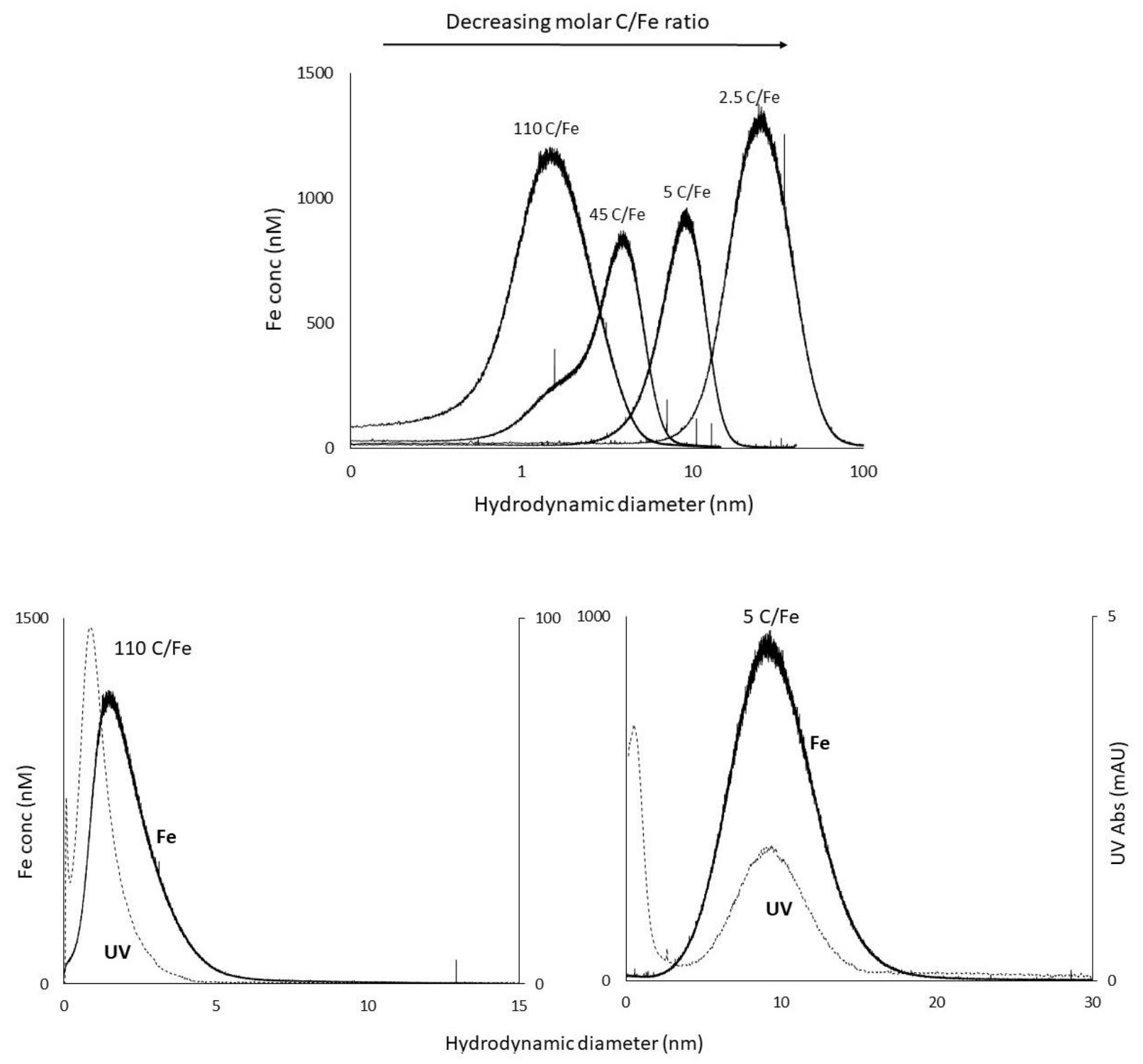

Figure 2 Top: FIFFF fractograms from HFO particles showing that Fe particle size increases with decreasing humics content in the samples (decreasing $\mathrm{C} /$ Fe ratio). Bottom: fractogram of Fe and UV of high (left) and low (right) $\mathrm{C} / \mathrm{Fe}$ of the HFO particle. At high C/Fe ratio, Fe forms complexes with the humic susbtances because both Fe and absorbance peak coincide at small particle size $(<5 \mathrm{~nm})$ whereas in the lower $\mathrm{C} / \mathrm{Fe}$ sample, HFO particles are formed of about $10 \mathrm{~nm}$ with adsorbed humic substances. 


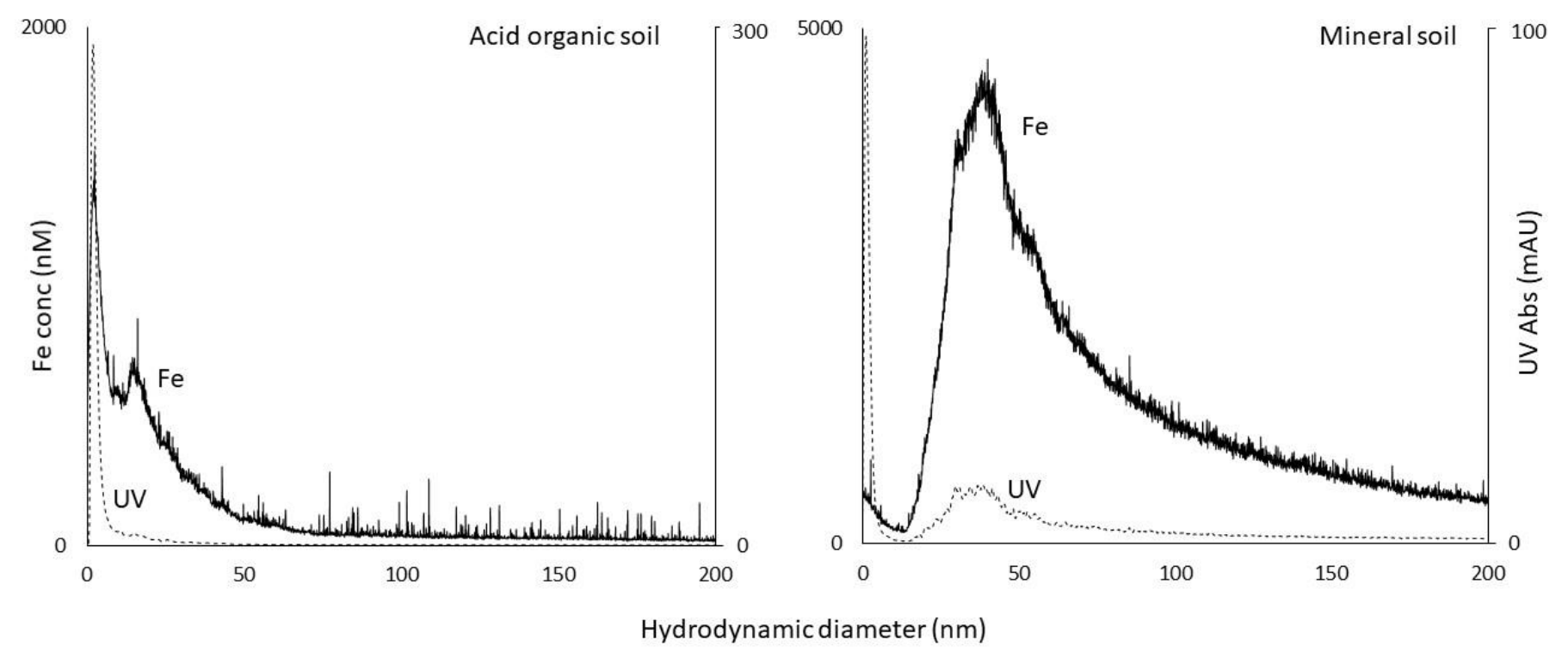

Figure 3 FIFFF fractograms of the soil solutions showing small ( $30 \mathrm{~nm})$ Fe particles in the acid, organic soil (left) and larger (30-200 nm) particles in the mineral soil (right). 


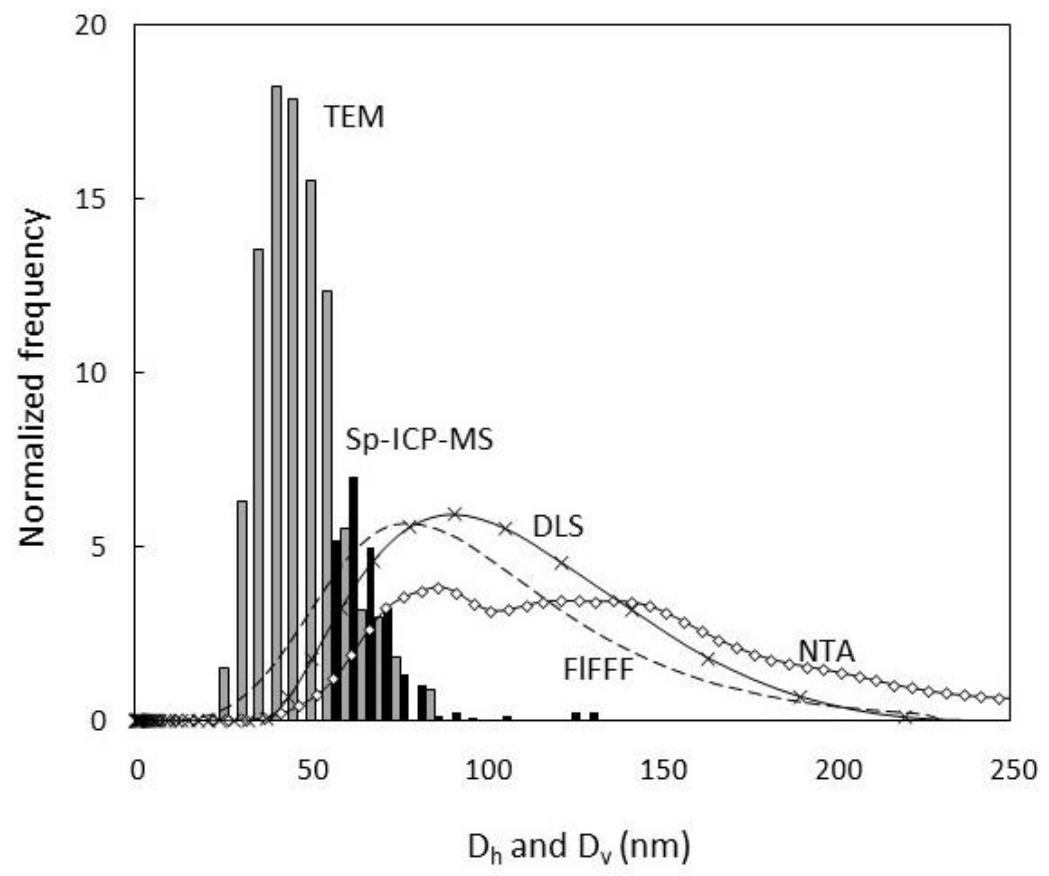

Figure 4 Overview of mass-based particle size distributions for Gt-NOM colloids with hydrodynamic diameter $D_{h}$ obtained with FIFFF, DLS and NTA and spherical volume equivalent diameter $D_{v}$ obtained with sp-ICP-MS analysis and TEM. The size distributions from TEM, NTA and sp-ICP-MS are calculated from the originally obtained number-based size distributions using particle density of $4 \mathrm{~g} \mathrm{~cm}^{-3}$. The mass-based spICP-MS data were additionally corrected for Fe recovery because the low Fe recovery $(26 \pm 4 \%)$ was attributed to particles below the size detection limit. The size distributions from TEM, sp-ICP-MS, FIFFF and NTA are plotted with equal bin size. 


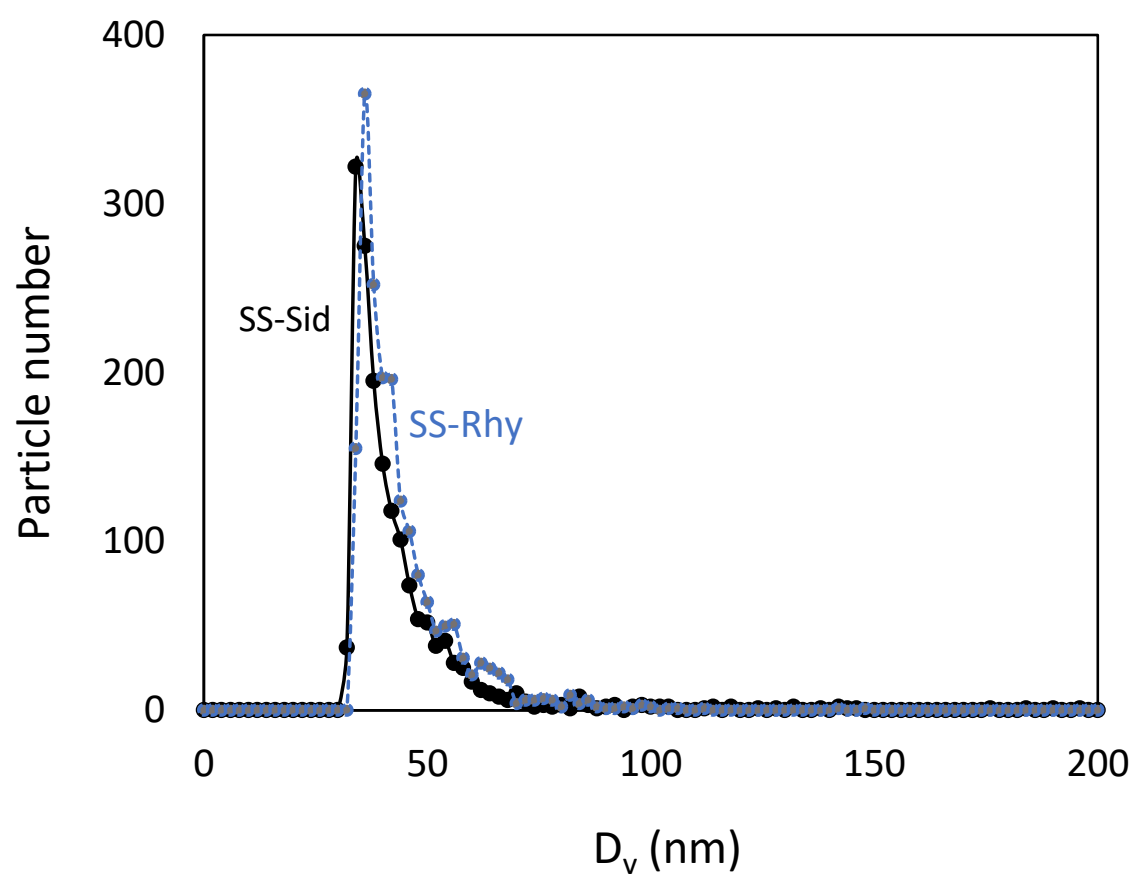

Figure 5 Spherical volume equivalent diameter $\left(D_{v}\right)$ with corresponding particle numbers in the soil solutions obtained with sp-ICP-MS. The size distribution is very similar but the Fe mass recovery is higher in the soil solution from the acid, organic soil (SS-Rhy) compared to the mineral soil (SS-Sid), respectively $45 \pm 11$ and $31 \pm 3 \%$. 